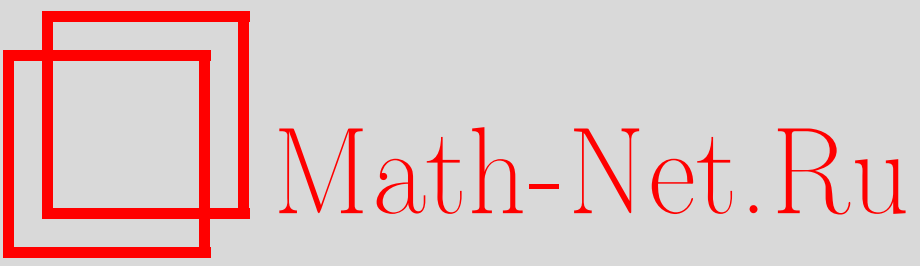

Л. О. Чехов, Спектральная задача на графах и $L$-функции, УMH, 1999, том 54, выпуск 6, 109-148

DOI: https://doi.org/10.4213/rm231

Использование Общероссийского математического портала Math-Net.Ru подразумевает, что вы прочитали и согласны с пользовательским соглашением

http://www.mathnet.ru/rus/agreement

Параметры загрузки:

IP: 54.166 .219 .16

26 апреля 2023 г., 13:33:44 


\title{
СПЕКТРАЛЬНАЯ ЗАДАЧА НА ГРАФАХ И L-ФУНКЦИИ
}

\author{
Л.О. ЧЕХОВ
}

\begin{abstract}
Исследуется процесс рассеяния на многопетлевых однородных $(p+1)$-валентных графрах (обобщенных деревьях). Эти графы суть одномерные связные симплициальные комплексы - фактор-графы однородного дерева относительно дискретно действующих подгрупп проективной группы $P G L\left(2, \mathbb{Q}_{p}\right)$. Как однородные пространства эти граффы идентичны $p$-адическим многопетлевым поверхностям. С конечным подграфом - редуцированным графом, включающим в себя все петли обобщенного дерева, ассоциируется $L$-функция Ихары-Сельберга. Изучается спектральная задача и вводится специальное понятие сферических функций - собственных функций дискретного оператора Лапласа, действующего на соответствующем графе. Определяется $S$-матрица и доказывается ее унитарность. Также приводится доказательство теоремы Хашимото-Басса, которая выражает $L$-функцию произвольного конечного (редуцированного) графа через детерминант локального оператора $\Delta(u)$, действующего на этом графе; детерминант $S$-матрицы выражается через отношение $L$-функций, таким образом задавая аналог формулы следа Сельберга. Точки дискретного спектра определяются и классифицируются с помошью $L$-функции. Приводятся примеры $L$-функциональных вычислений.
\end{abstract}

Библиография: 47 названий.

\section{СОДЕРЖАНИЕ}

Введение

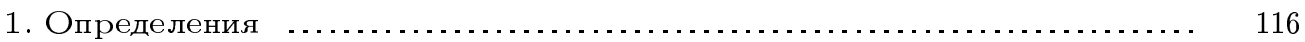

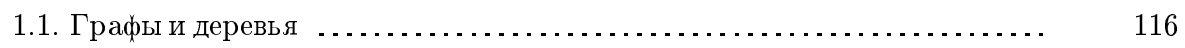

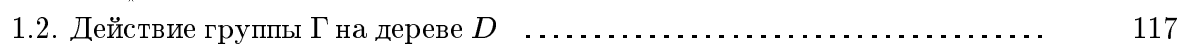

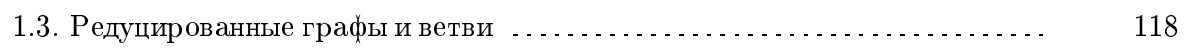

2. Функции, операторы и потенциалы на графах .................... 119

2.1. Автоморфные функции и потенциалы на $T$....................... 119

2.2. Операторы Гекке на граф̆ах ................................ 121

2.3. Операторы смешивания между пространствами $C_{0}$ и $C_{1} \ldots \ldots \ldots \ldots . .2124$

2.4. Соотношения между операторами ............................. 124

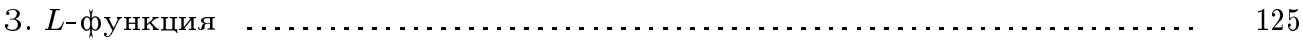

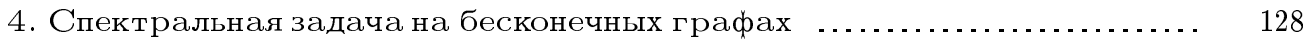

4.1. Сферические функции на факторизованных деревьях . . . . . . . . . . . . 130

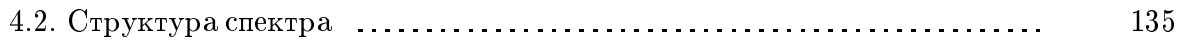




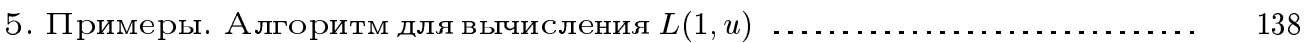

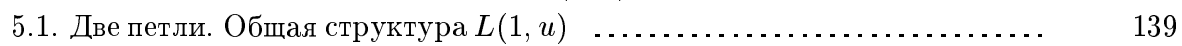

6. Пространства Тейхмюллера и графы …........................ 140

6.1. Формула следа Сельберга и распределение геодезических . . . . . . . . . . . 143

6.2. Классические проективные (модулярные) преобразования ........... 145

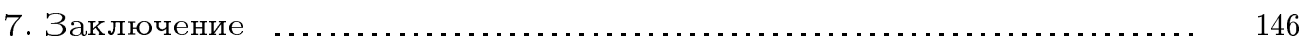

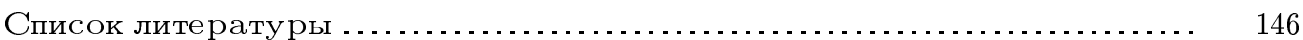

\section{Введение}

Два фундаментальных результата, связьвающие $L$-функции (дзета-функции) с детерминантами Лапласа, были получены Сельбергом [1] (см. также [2]-[4]) (и развиты впоследствии в работах П. Лакса и Р. Филлипса [5], Л. Д. Фаддеева и Б. С. Павлова [6], А.Б. Венкова [7], и др.) и И. Ихарой [8], К.-И. Хашимото [9], [10] и Х. Бассом [11]. Первьй результат - знаменитая формула следа Сельберга - связывает дзета-функции и детерминанты Лапласа на компактных (или некомпактных с конечной площадью) в общем случае многомерных ( $D$-мерных) многообразиях постоянной отрицательной кривизны, которые могут быть получены из $D$-мерного верхнего полупространства с гиперболической метрикой путем факторизации по дискретно действующей подгруппе группы симметрий этого пространства. Эти результаты вкратце обсуждаются ниже в связи с результатами, относяшимися к $L$-функциям Ихары-Сельберга на конечных графах.

Дзета-функция конечного графа была впервые введена Ихарой [8] при доказательстве структурной теоремы для свободных от кручения дискретных кокомпактных подгрупп Г группы $P G L\left(2, \mathbf{K}_{p}\right)$; при этом $\mathbf{K}_{p}$ - это $p$-адическое числовое поле, или поле степенных последовательностей над конечным полем. Группа Г действует свободно, и ассоциированная дзета-функция строится следующим образом. Элемент $\gamma \in \Gamma, \gamma \neq 1$, назьвается примитивным әлементом, если он полностью порождает свою стабилизирующую подгруппу в Г. Определим длину $l(\gamma) \equiv\left\|\lambda_{1} / \lambda_{2}\right\|_{p}$ элемента, где $\|\cdot\|_{p}-$ норма в $\mathbf{K}_{p}$ и $\lambda_{1}, \lambda_{2} \in \mathbf{K}_{p}$ - собственные значения $\gamma$. Пусть $\mathscr{P}(\Gamma)$ - множество классов сопряженности примитивных элементов группы Г. Тогда дзета-функция Ихары имеет вид:

$$
Z_{\Gamma}(u)=\prod_{\gamma \in \mathscr{P}(\Gamma)}\left(1-u^{l(\gamma)}\right)^{-1}
$$

Это определение было расширено на конечномерные представления группы Г в работе [12]; в этом случае вместо (0.1) получим:

$$
Z_{\Gamma}(u, \rho)=\prod_{\gamma \in \mathscr{P}(\Gamma)} \operatorname{det}\left(I_{1}-u^{l(\gamma)} \rho(\gamma)\right)^{-1}
$$

где $\rho(\gamma)$ - характер данного представления, а $I_{1}$ - единичный оператор. (Исходная формула (0.1) отвечает случаю тривиального представления $\rho(\gamma) \equiv 1$.)

Работа выполнена при поддержке Российского фонда фундаментальных исследований (грант № 98-01-00327). 
Хотя дзета-функция (0.2) и представляется в виде бесконечного произведения, она оказывается рациональной функцией. Теорема Ихары утверждает, что

$$
Z_{\Gamma}(u, \rho)=\left(1-u^{2}\right)^{s} \operatorname{det}\left(I_{0}-A u+p u^{2}\right),
$$

где $s$ - целое число, а $A$ - матрица, действуюшая в конечномерном линейном пространстве ( $I_{0}$ - единичная матрица этого пространства). Используя формулу рациональности, можно найти число классов сопряженности примитивных элементов $\Gamma$.

1. В работах [13], [14] исследовались процессы рассеяния на бесконечных граффах, представляюших собой пространства постоянной отрицательной кривизны. Это - однородные, или унивалентные графы, для которых существует такое натуральное число $p \geqslant 1$, что все вершины графа иншидентны в точности $p+1$ концу ребер графа. Если число $p$ простое, то такие графы интерпретируются как однородные пространства $p$-адической проективной группы $P G L\left(2, \mathbb{Q}_{p}\right)$, факторизованные, во-первых, по ее максимальной компактной подгруппе $P G L\left(2, \mathbb{Z}_{p}\right)$ и, во-вторых, по некоторой дискретной свободно действуюшей группе Г (группе Шоттки). Тогда однородное пространство $D \simeq P G L\left(2, \mathbb{Q}_{p}\right) / P G L\left(2, \mathbb{Z}_{p}\right)$ - это однородный бесконечный древесный граф. Если после этого профакторизовать дерево $D$ по некоторой свободно действующей конечнопорожденной подгруппе $\Gamma_{g} \subset P G L\left(2, \mathbb{Q}_{p}\right)$, где $g$ - число производящих элементов, то полученный граф $T_{g}=D / \Gamma_{g}$ снова будет однородным графом, содержашим теперь $g$ петель; дерево $D$ при этом есть универсальная накрываюшая полученного графа.

В статье приводится аналог формулы следа Сельберга [1] для таких дискретных поверхностей. Эти графы имеют, в частности, смысл многопетлевых $p$-адических поверхностей, поскольку в работах [15]-[18] было показано, что для простых $p$ действие дискретного лапласиана на таких графах задает правильные амплитуды рассеяния $p$-адических струн.

Заметим, однако, что вычисления в подходе $p$-адических струн проводились лишь для тривиального (абелева) представления групшы Г. Естественное расстояние $\left|P_{x, y}\right|$ вдоль пути $P_{x, y}$, соединяюшего точки графа, - это просто число ребер, образуюших (с кратностями) этот путь. Удобно рассматривать линейные пространства функций $C_{0}$ и $C_{1}$, зависяших соответственно от вершин $x_{i}$ и ориентированных ребер $\vec{e}_{j}$ графа $T$. Оператор Лапласа $\Delta$ действует на пространстве $C_{0}$ обычным образом:

$$
\Delta f(x)=\sum_{\left|P_{x, x_{i}}\right|=1} f\left(x_{i}\right)-(p+1) f(x) .
$$

Удобно выделить из графа $T$ некоторый конечный “замкнутый” подграф - редуцированны й граф $F$, который связен и содержит все петли исходного графа. Валентности вершин графа $F$ теперь могут быть произвольньми (не превьшшаюшими $p+1)$. В работе [18] была предложена формулировка $p$-адических обобщений всех основных понятий теории (открытых) струн таких, как формы Прима, группы Шоттки, и т. д., что позволило найти соответствуюшие амплитуды рассеяния.

2. Формула следа Сельберга, которая устанавливает явное соотношение между детерминантами операторов Лапласа и дзета-функциями (или же $L$-функциями Ихары-Сельберга), в случае обычных замкнутых (или, в более общем случае, имеюших 
конечную площадь или объем) римановых поверхностей (многообразий) действительно близка по виду к (0.3). Расстояние $l(\gamma)$ в данном случае - это длина замкнутой геодезической в метрике постоянной отрищательной кривизны. Однако не существует подходящего аналога формулы Сельберга для существенно некомпактного случая, когда спектр лапласиана содержит непрерьвную часть, отвечающую рассеянию. Заметим, однако, что дзета-функция (0.1) остается хорошо определенной и в случае таких некомпактных поверхностей; именно проблемы с определением спектра оператора Лапласа не позволяют найти аналогичное соотношение. В этой связи можно ожидать, что описание пространств модулей комплексных кривых с дырками с помошью графов [19]-[22], которое тесно связано с описанием в терминах геодезических, может привести к новым комбинаторным результатам для некомпактных римановых поверхностей. Этот подход обсуждается в разделе 6.

3. По соглашению считается, что дзета-функции принимают значения в поле $\mathbf{k}_{p}$ (определение соответствуюших некоммутативных детерминантов содержится в [11]), в то время, как $L$-функции принимают значения в поле комплексных чисел $\mathbb{C}$.

В цикле элегантных работ К.-И. Хашимото [9], [10] и Х. Басса [11], посвященных исследованиям дзета- и $L$-функций, формула Ихары была обобщена на случай произвольного (не обязательно унивалентного) конечного графа. В наиболее общем виде она вьглядит следующим образом:

$$
L(u, \rho)=\operatorname{det}^{-1}\left(I_{1}-u T_{1}\right),
$$

где новый оператор $T_{1}$ - это оператор, действующий на пространстве функций от ориентированных ребер (симплексов размерности один) и соответствующий переносу вдоль ребер граф̆а.

Соотношение (0.4) выполнено как для дзета-, так и для $L$-функций; различны лишь определения детерминантов (коммутативных или некоммутативных), входящих в это соотношение. В настоящей статье рассматривается только случай $L$-функций, которьй представляет физический интерес с точки зрения спектральной задачи и задачи рассеяния на графах с потенциалами.

Формула (0.4), оказьвается, замечательным образом универсальна; она выражает условие рациональности, которому дзета-функции удовлетворяют в громадном числе случаев, относящихся к дискретной динамике, - указанные соотношения обобшаются на случай эрмитовых (неабелевых) потенщиалов шрёдингеровского типа, заданных на ребрах графа [23], [24], на случай ненулевого кручения (т.е. для потенциалов, зависящих от "углов" - от пар последовательных ребер $\left.\left(\vec{e}_{i}, \vec{e}_{i+1}\right)\right)$ [25], [26], и т. д. Однако, в большинстве этих случаев не сушествует явного способа связать соотношение (0.4) с детерминантами соответствующих операторов Лапласа на неоднородных графах. Только лишь в случае, когда потенциал $U_{\vec{\mu}}$ на (ориентированных) ребрах удовлетворяет условию унитарности, т.е. $U_{\vec{\mu}}=U_{\overleftarrow{\mu}}^{-1}$ для любой пары противоположно ориентированных ребер, $L$-функция может быть выражена через детерминанты лапласовского типа на произвольных (не обязательно унивалентных) графах:

$$
L(u, \rho)=\left(1-u^{2}\right)^{s} \operatorname{det}^{-1}\left(1-u M_{1}+u^{2} Q\right) \equiv\left(1-u^{2}\right)^{s} \operatorname{det}^{-1} \Delta(u),
$$


где $M_{1}$ - вышеупомянутьй оператор суммирования по всем соседним вершинам (возможно зависящий от потенциала), а новьй по сравнению с (0.2) элемент - это оператор $Q$, которьй вычисляет число соседних вершин: $Q x=q x$, если у вершины $x \in T$ имеется $q+1$ соседних вершин. В случае тривиального (одномерного) представления $s=|V|-|E|$, где $|V|$ и $|E|$ - соответствующие полные числа вершин и (неориентированных) ребер графа (в неабелевом случае $s$ - это разность полных размерностей линейных пространств $v_{\mathscr{A}}^{|V|}$ и $v_{\mathscr{A}}^{|E|}$, где предполагается, что на ребрах и в вершинах граф̆а задаются векторы представления $v_{\mathscr{A}}$ групшы или алгебры $\left.\mathscr{A}\right)$. Заметим, что оператор $\Delta(u)$ становится лапласианом только при $u=1$; эти операторы даже не коммутируют при различных $u$. (Описание этих результатов и различных их применений содержится в обзоре [27].)

4. Графы $T$ ( $p$-адические многопетлевые поверхности) в общем случае некомпактны, что отличает их как от замкнутых римановых поверхностей, так и от конечных графов. Оказывается, однако, что в этом случае можно использовать технику сферических функций и описать задачу в физических терминах теории рассеяния на графах. В первоначальном подходе сферические функции $\psi$ - это те собственные функции оператора Бельтрами-Лапласа, которые зависят только от расстояния до выделенной точки $x_{0}$ (центра). Поскольку лапласиан - оператор второго порядка, каждое такое решение на асимптотике имеет две экспоненциальные ветви, пропорциональные $\alpha_{+}^{d}$ и $\alpha_{-}^{d}$, где $d$ - расстояние до центра. При разрешении задачи на собственные значения в центральной точке фиксируется отношение коэффициентов $a_{+}$и $a_{-}$, стоящих при этих двух ветвях решения. Выбирая $\psi\left(x_{0}\right)=1$, получим $a_{+}$и $a_{-}$в виде так назьваемых коэффициентов Хариш-Чандра, а $c=a_{+} / a_{-}$при этом задает амплитуду рассеяния $s$-волны. Сферические функции в дискретных пространствах были найдены в задаче рассеяния на квантовой гиперболической плоскости [28], равно как и в задаче рассеяния на $p$-адической гиперболической плоскости [29]. Полученные $S$-матрицы оказываются тесно связанными со статистическими функциями $X X Z$-модели, для которых П. Фройнд и А. В. Забродин получили цельй набор красивых, но во многом до сих пор не понятых соотношений (см. [30] и библиографию там).

Исследование спектральной теории на графах имеет уже достаточно долгую и богатую историю. Полученные результаты в основном относились к исследованию проблем на собственные значения для конечных однородных (т.е. унивалентных) графов. Была прослежена глубокая связь меж ду теорией модулярных форм на пространствах Тейхмюллера и конечными графами, а именно, графами Рамануджана. Этот интересный подход не будет обсуждаться в данной работе, отметим лишш, что его исследование можно найти в книге [31] и работах [32], [33], [27].

В работе [13] был предложен аналог сферической функции для многопетлевого графа. Главная идея этого подхода состоит в том, что если рассмотреть на графе спектральную проблему $L \psi=\lambda \psi$, где $L$ - лапласиан, то в силу линейности задачи любая линейная суперпозищия сферических функций, отвечающих одному и тому же собственному значению $\lambda$, но имеюших различные "рассеивающие центры", опять есть собственная функция. Собственная функция оператора Лапласа на факторизованном дереве $T=X / \Gamma_{g}$ отвечает периодической функции распределения источников $s(x)$ на дереве $X$, т.е. для каждого элемента $\gamma \in \Gamma_{g}$ и $x \in X$ имеем $s(\gamma x)=s(x)$. Тогда и вся собственная функция оказывается периодичной относительно действия груп- 
пы $\Gamma_{g}$. Далее, можно выбрать конечный подграф (редуцированньй граф̆) $F \subset T$ и рассматривать только такие $s(x)$, для которых $\operatorname{supp} s(x) \subseteq F$. Заметим, что внутри $T$ имеется единственньй минимальный конечньй редуцированньй подграф, содержащий все петли, - объединение $D(\Gamma)$ инвариантных осей всех элементов группы $\Gamma_{g}$, профакторизованное по действию самой групшы $\Gamma_{g}$. Этот графф $D(\Gamma) / \Gamma$ содержит всю информацию о “геометрической структуре" графа $T$. В дальнейшем всегда предполагается, что $D(\Gamma) / \Gamma \subseteq F$.

Каждая собственная функция $\psi(x)$ представима в виде суммы падающей и отраженной волн:

$$
\psi(x)=A_{\mathrm{adv}}(u) \alpha_{+}^{d(x, u(x))}-A_{\mathrm{ret}}(u) \alpha_{-}^{d(x, u(x))} \equiv \psi_{+}(x)-\psi_{-}(x),
$$

где $\alpha_{+}$и $\alpha_{-}-$два фиксированных комплексных числа, зависяших только от собственного значения $t$ оператора Лапласа $L \equiv \Delta(1)$, действуюшего на полном графе $T$, $L \psi=(t-p-1) \psi$, и от исходного числа инцидентности $p, \alpha_{ \pm}=\frac{t}{2 p} \pm \sqrt{\frac{t^{2}}{4 p^{2}}-\frac{1}{p}}$, $\alpha_{+} \alpha_{-}=1 / p ; u(x)$ - это ближайшая к точке $x$ точка редуцированного граффа $F$ (если $x \in F$, то $u(x)=x)$. Как и в центрально-симметричном случае, функция $\psi(x)$ на внешних ветвях графа $F$ зависит только от расстояния до $F$.

Для таких систем можно определить матрицу рассеяния $S$. Выберем базисные функции $A_{\mathrm{adv}}^{i}(u)$ и $A_{\mathrm{ret}}^{i}(u)$, которые отличаются от нуля только в некоторой $(i$-й $)$ точке $F$. Можно определить асимптотические векторы $\psi_{i}^{ \pm}$, которые пропорциональны $\alpha_{ \pm}^{n}$ при движении вдоль $i$-й ветви и равны нулю на других ветвях. После этого можно асимптотически разложить $\psi$ в сумму базисных векторов: $\psi_{i_{a}}^{+}-\sum_{\left\{j_{b}\right\}} s_{i_{a}, j_{b}} \psi_{j}^{-}$, где $i_{a}, j_{b}$ - мультииндексы, включаюшие в себя также индексы компонент $a$ представления $v_{\mathscr{A}}$ на $i$-й ветви. Тогда матрица $s_{i_{a}, j_{b}}$ имеет естественньй смысл $S$-матрицы рассеяния.

Детерминант матрищы $S$ зависит от спектрального параметра, содержит информацию о спектре задачи и, кроме того, напрямую связан с $L$-функцией графа $T$. (Описание собственных функций в рамках подхода Лакса и Филлипса было дано в работе [34].)

Чтобы найти детерминант матрицы $S$, можно наложить ограничение: $\frac{A_{\mathrm{adv}}(u)}{A_{\mathrm{ret}}(u)}=$ const для всех - как внешних, так и внутренних - точек $u \in F$. Наложение этого условия во всех точках подграфқа $F$ позволяет избавиться от неоднозначности в расщеплении собственной функции $\psi(x)$ на падающую и отраженную волны.

Поскольку центральньй объект конструкции - редуцированньй граф $F$ - конечен, то конечно и число собственных значений $S$-матрицы, т.е. всех возможных констант $c_{i}$ из предыдушего условия (само обозначение " $c$ " напоминает о своем происхождении из $c$-функции Хариш-Чандры [35]). Произведение этих констант, обозначаемое как $C$, таким образом является детерминантом $S$-матрищы, также называемым полной $C$-функцией. Ниже дается доказательство основной формулы, устанавливающей связь между величиной $C$ и детерминантом локального оператора $\widetilde{\Delta}(u)$, действующего только на редуцированном графе $F$ :

$$
\operatorname{det} S(t)=\left(\frac{\alpha_{+}}{\alpha_{-}}\right)^{r_{0}} \frac{\operatorname{det} \widetilde{\Delta}\left(\alpha_{-}\right)}{\operatorname{det} \widetilde{\Delta}\left(\alpha_{+}\right)},
$$


где $r_{0}$ - полная размерность линейного пространства функций, заданных в вершинах редуцированного графа $F$ и принимающих значения в представлении $v_{\mathscr{A}}$.

Напротив, оператор $\widetilde{\Delta}\left(\alpha_{\mp}\right)$ в формуле $(0.7)$ берется из $(0.5), \widetilde{\Delta}(u)=1+\widetilde{Q} u^{2}-u \widetilde{M}_{1}$ (величины с волной - операторы, действующие на функциональных подпространствах, ассоциированных с редуцированньм графом), и он полностью определяется в терминах самого редуцированного графа; зависимость от “большого" графа $T$ содержится лишш в аргументах $\alpha_{+}$и $\alpha_{-}$функции $\widetilde{\Delta}\left(\alpha_{ \pm}\right)$. Сравнивая выражения $(0.7)$ и $(0.5)$, можно получить соотношение между полной $C$-функцией и $L$-функцией $p$-адической кривой:

$$
C=\left(\frac{\alpha_{+}}{\alpha_{-}}\right)^{r_{0}}\left(\frac{1-\alpha_{-}^{2}}{1-\alpha_{+}^{2}}\right)^{r_{0}-r_{1}} \frac{L\left(\alpha_{+}\right)}{L\left(\alpha_{-}\right)}
$$

где $r_{0}-r_{1}-$ это разность полных размерностей пространств функций, заданных в вершинах и на (неориентированных) ребрах; она равна $(g-1) \times\left|v_{\mathscr{A}}\right|$, где $g$ - число петель (род) графа, а $\left|v_{\mathscr{A}}\right|$ - размерность представления. С помошью геометрического подхода работы [24] можно доказать, что $S$-матрица унитарна в зоне рассеяния.

С другой точки зрения, подходящие произведения коэффициентов рассеяния при разных $p$ соответствуют адельной идеологии. Использовать такие произведения для описания процессов рассеяния было предложено в работе [29], в которой произведение $C$-функций, отвечаюших рассеянию на $p$-адических гиперплоскостях, взятое по всем простьм $p$, оказалось связанным с $C$-функцией рассеяния на модулярной фигуре рода один - процесс, которьй рассматривался Л. Д. Фаддеевым и Б. С. Павловым [6]. Наиболее обшие формулы для рассеяния на симметрических пространствах были получены в [36] с помощью техники гамма-фрунции. Можно надеяться найти подходящее адельное произведение $L$-функций, возникающих в данном подходе, чтобы сравнить его с подобными произведениями для римановых поверхностей.

Также обсуждается задача на собственные значения, относящиеся к дискретной части спектра оператора Лапласа, и доказываются два важных соотношения, которым удовлетворяет $S$-матрица. Дискретная составляющая спектра может содержать помимо "стандартного" дискретного спектра, собственные значения которого отвечают экспоненциально спадаюшим на ветвях графа собственным функциям, также так называемые исключительные собственные значения, отвечаюшие таким собственным функциям, которые в точности равны нулю на всех ветвях. Сначала можно доказать, что полюсы $L$-функции отвечают нормальным дискретным собственньм значениям тогда и только тогда, когда эти полюсы не сокращаются в формуле $(0.8)$, т.е. если $\alpha_{+}$ и $\alpha$ - не являются одновременно полюсами $L$-функции. Напротив, если такое сокрашение происходит, т.е. сушествуют такие полюсы $\alpha_{+}$и $\alpha_{-} L$-функции, что $\alpha_{+} \alpha_{-}=1 / p$, то в задаче возникает исключительньй дискретньй спектр.

Работа имеет следуюшую структуру. В разделе 1 собраны определения, интерпретация $p$-адических многопетлевых поверхностей как графов и описано действие группы Шоттки на исходном однородном дереве. В разделе 2 описываются автоморфные функции и потенциалы на таких графах и вводятся операторы, действующие на клеточных комплексах, связанных с обобщенными деревьями. В разделе 3 вводятся 
$L$-функции, ассоциированные с такими группами, или, эквивалентно, с редуцированными графами. В этом разделе также формулируется и доказьвается теорема Хашшмото-Басса. В разделе 4 рассматривается спектральная задача, вводятся сферические функции на многопетлевых графах, определяются соответствующие $S$-матрицы и показьвается, что их детерминанты выражаются через отношение двух $L$-функций. Также в этом разделе доказьвается унитарность $S$-матрицы и представлен рецепт нахождения собственных значений из дискретного спектра с помощью техники $L$-функции. В разделе 5 даются примеры явных ответов для рода 1 и 2 и приводится простой алгоритм для выгисления $L$-функций в низших родах с помошью теоремы Хашимото-Басса. Наконец, в разделе 6 дается конструкция графов, описьвающих пространства Тейхмюллера комплексных кривых в униформизации Пуанкаре, и ставится проблема найти подходящий аналог формулы следа Сельберга для открытых римановых поверхностей.

\section{1. Определения}

1.1. Графы и деревья. Пусть $p$ - натуральное число и $D$ - однородный графф - есть дерево порядка $p+1, V(D)$ и $\vec{L}(D)$ - соответствуюшие множества его вершин и (ориентированных) ребер. В дальнейшем рассматриваются ориентированные ребра, т.е. каждому неориентированному ребру отвечает двумерное линейное подпространство в $\vec{L}(D)$, базисные векторы которого отвечают двум возможным ориентациям данного ребра.

Pacстояние $d(x, y)$ между двумя точками $x, y$ на дереве равно длине единственного пути, связывающего эти две точки.

ОПРЕДЕЛЕНИЕ 1. Пусть $T$ - граф̆ с конечным числом петель и ветвей (хвостов), $V$ - множество его вершин и $\vec{L}$ - множество (ориентированных) ребер. Граф $T$ - это фактор своего универсального накрьвающего графа (дерева) $D$ относительно действия свободно действуюшей подгрупшы Г группы движений дерева, т.е. $T=D / \Gamma$. Обозначим через $\vec{e}$ и $\overleftarrow{e}$ два элемента из $\vec{L}$, отвечающие одному и тому же ребру с противоположными ориентациями. Модуль (абсолютное значение) $|\cdot|$ множества это количество элементов (возможно, бесконечное) этого множества.

ОПРЕДЕЛЕНИЕ 2. Ориентированный $n y m b P_{x, y}$ в графе $T$ (или $\left.D\right)$ - это (единственная) последовательность (конечная или бесконечная) $\left(\vec{e}_{1}, \ldots, \vec{e}_{m}\right)$ последовательных $\left(d_{0} \vec{e}_{i}=d_{1} \vec{e}_{i-1}, 1<i \leqslant m\right.$, где $d_{0}$ и $d_{1}-$ соответствующие операторы, проецирующие ориентированное ребро на начальную или конечную вершину) ориентированных ребер без возврашений (т.е. $\vec{e}_{i} \neq \overleftarrow{e}_{i-1}$ при $1<i \leqslant m$ ), начинаюшаяся в вершине $x=d_{0} \vec{e}_{1}$ и оканчивающаяся в вершине $y=d_{1} \vec{e}_{m}$. Длина nути $\left|P_{x, y}\right|-$ это полное число ребер (с учетом кратности), входящих в этот путь. На дереве $D$ длина пути $P_{x, y}$ всегда равна расстоянию между вершинами $x$ и $y$.

Путь $P_{x, y}$ назьвается замкнутым, если $x=y$. Собственный замкнуты й путьэто замкнутый путь, в котором $\vec{e}_{1} \neq \overleftarrow{e}_{m}$ (для путей длины больше единищы).

Ребро $\vec{e}_{\mu}$ называется следующим за ребром $\vec{e}$, если $\vec{e}_{\mu}$ начинается в вершине, в которой $\vec{e}$ оканчивается, т.е. $d_{0} \vec{e}_{\mu}=d_{1} \vec{e}$. 
ЗАмечание 1. Множество собственных замкнутых путей, в которых начальная (= конечная) точка не указывается (что достигается с помошью "забываюшего" отображения), находится во взаимно однозначном соответствии с классами сопряжений гомотопической группы $\pi_{1}(T)$.

1.2. Действие группы $Г$ на дереве $D$. Напомним некоторые конструкции, возникаюшие при действии свободно действуюшей конечнопорожденной группы $\Gamma$ на дереве $D$.

Бесконечное дерево $D$ обладает богатой группой изометрий - преобразований, сохраняюших расстояние на графе. Рассматривая “жесткий” граф, т.е. граф с фиксированным порядком ребер, входящих в каждую вершину, и накладывая условие сохранения этого порядка при действии группы симметрий, можно редуцировать эту большую группу к группе врашений и сдвигов вдоль осей (бесконечных путей без возврашений) в дереве. (Гранища дерева $D$ представима как $p$-адическая проективная прямая $P_{1}\left(\mathbb{Q}_{p}\right)[15]$. Тогда полная группа движений дерева $X$ - это проективная группа $P G L\left(2, \mathbb{Q}_{p}\right)$.) Элемент, который задает врашение (и аналогичен, тем самьм, эллиптическому элементу обычной проективной групш) - это вращение дерева как целого вокруг вершины $x_{0} \in D$ на угол $2 \pi n /(p+1), 1 \leqslant n \leqslant p$. В этой статье такие элементы не рассматриваются. Другой вид преобразований задается элементами, не имеюшими неподвижных точек внутри дерева (такие элементы называются свободно действуюшими).

Без ограничения обшности считаем группу $Г$ дискретной свободно действуюшей конечнопорожденной подгруппой $P G L\left(2, \mathbb{Q}_{p}\right)$, т.е. внутри дерева не существует ни одной точки, остаюшейся на месте при действии какого-либо элемента этой групшы, отличного от единичного. (Тем самым, эта группа - аналог фуксовой группы в непрерьвной гиперболической геометрии.) В дальнейшем рассматриваются только конечнопорожденные группы $\Gamma$, которые задаются $g$ производящими элементами. Каждый элемент $\gamma \in \Gamma, \gamma \neq 1$, определяет перенос (сдвиг) дерева как целого вдоль инвариантной оси $D(\gamma) \subset D$ данного элемента. Инвариантная ось $D(\gamma)$ - это единственный бесконечный ориентированньй путь $\ldots \vec{e}_{-1} \vec{e}_{0} \vec{e}_{1} \vec{e}_{2} \ldots$, который отображается в себя под действием элемента $\gamma: \gamma\left(\vec{e}_{i}\right)=\vec{e}_{i+l}$, при этом сдвигаясь в положительном направлении относительно своей ориентации. Здесь $l \equiv l(\gamma)-$ длина әлемента, $l(\gamma)=\inf \{d(x, \gamma(x)) \mid x \in V(D)\}$, где минимум очевидно достигается на множестве $x \in D(\gamma)$. Таким образом, элемент $\gamma$ задает сдвиг на расстояние $l(\gamma)$ вдоль прямой (оси) $D(\gamma)$. (В дальнейшем предполагается, что $l(\gamma)<\infty$. $)^{1}$

Стабилизатор $Z(\gamma) \subset$ Г элемента $\gamma \in \Gamma$ - это циклическая группа. Если, кроме того, сам элемент $\gamma$ порождает группу $Z(\gamma)$, то $\gamma$ назьвается примитивным әлементом группы Г. Удобно определить функцию фон Мангольта $\Lambda(\gamma)=l(\varpi)$, где $\gamma=\varpi^{m}$ и $\varpi-$ примитивный элемент $\Gamma$.

Рассмотрим теперь поддерево $D(\Gamma)$, являющееся объединением всех инвариантных осей элементов группы $\Gamma$. Если элемент $\gamma$ имеет длину $l(\gamma)$, то последовательность $\ldots \vec{e}_{-1} \vec{e}_{0} \vec{e}_{1} \vec{e}_{2} \ldots$ имеет структуру $\ldots\left(\vec{e}_{1} \vec{e}_{2} \ldots \vec{e}_{l}\right)\left(\vec{e}_{1}^{\prime} \vec{e}_{2}^{\prime} \ldots \vec{e}_{l}^{\prime}\right)\left(\vec{e}_{1}^{\prime \prime} \vec{e}_{2}^{\prime \prime} \ldots \vec{e}_{l}^{\prime \prime}\right) \ldots$ где $\vec{e}_{i}^{(n)}-$ копии ребра $\vec{e}_{i} \in T$. Более того, последовательность $\vec{e}_{1} \vec{e}_{2} \ldots \vec{e}_{l}$ должна

\footnotetext{
${ }^{1}$ Отметим, что в этой геометрии не существует прямого аналога параболического элемента.
} 
быть собственным замкнутым путем в графе $T$ (с возможными повторениями ребер, т.е. некоторые $\vec{e}_{i}$ могут иметь совпадаюшие прообразы в $\left.T\right)$.

Рассмотрим множество классов сопряженности $\{\gamma\}$ элементов групшы $\Gamma$ :

$$
\{\gamma\}:\left\{\bigcup_{\gamma, \omega \in \Gamma} \omega \gamma \omega^{-1} \in \Gamma\right\} .
$$

Для каждого элемента $\beta \in\{\gamma\}$ и каждой вершины $x \in V(D)$ получим:

$$
\beta x=\omega \gamma \omega^{-1} x=y
$$

следовательно, $\left(\omega^{-1} y\right)=\gamma\left(\omega^{-1} x\right)$. Таким образом, для каждого элемента $\beta \in\{\gamma\}$ существует такой $\omega \in \Gamma$, что инвариантная ось элемента $\beta$ есть образ инвариантной оси производящего элемента $\gamma$ при действии элемента $\omega^{-1}: D(\beta)=\omega^{-1} D(\gamma)$. Тем самым существует взаимно однозначное соответствие между классами сопряженности $\{\gamma\}$ и множеством всех собственных замкнутых путей в графе $T$.

Примитивнылми классами сопряженности $\{\varpi\}$ называются такие классы $\{\gamma\}$, которые порождаются примитивными элементами Г. При этом собственный замкнутый путь, соответствующий $\{\varpi\}$, - это собственньй замкнутый путь в $T$, который не содержит подпериодов.

Все графы, рассматриваемые в данной статье, получаются факторизацией однородного дерева $D$ относительно действия свободно действуюшей групшы симметрий.

\section{3. Редуцированные графы и ветви.}

ОПРЕДЕЛЕНИЕ 3. Пусть редуиированный граф $F$ - это конечньй, обязательно связньй подграф $F \subset T$, содержащий все петли графа $T$. Его универсальная накрывающая, $D_{F}$, есть поддерево в $D$.

Для общности полагается, что редуцированный граф̆ не обязан однозначно задаваться графом $T$. Тем не менее, всегда можно выделить минимальный редуцированный граф, являющийся пересечением всех возможных редуцированных графов, допускаемых данным графом $T$. Этот минимальньй редуцированньй графф в точности совпадает с объединением всех $D(\gamma), \gamma \in \Gamma$, профакторизованным по действию групшы Г. Это объединение есть поддерево $D(\Gamma) \subseteq D$, но ни $D(\Gamma)$, ни $F$ не обязаны быть однородными графоми.

Подграф̆ $D(\Gamma) / \Gamma$ и, следовательно, редуцированньй граф̆ $T$ - конечные графы, содержашие в точности $g$ петель, где $g$ (род) - число производящих элементов групшы Г.

Поскольку поддерево $D(\Gamma)$ в общем случае не совпадает с полным деревом $D$, фактор-граф $\Gamma \backslash X$ обычно бесконечен в отличие от графов в статье [8].

Граф $T$ может быть представлен в виде $T=F \cup B(T)$, где все $g$ петель графа $T$ содержатся в редуцированном графе $F$. Важная отличительная черта минимального редуцированного графа - это то, что он не содержит вершин, инцидентных только одному ребру из $F$. Дополнение к графу $F, B(T)$, - это (конечное либо пустое) множество ветвей, растуших из вершин $F$ таким образом, чтобы полная валентность каждой вершины графа $T$ была равна $p+1$. Всегда будет предполагаться, что полное число ветвей, растуших из вершины редуцированного графа, равно $p-q$, где $q+1-$ 


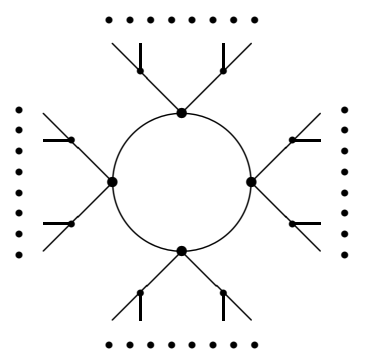

Рис. 1. Пример факторизованного дерева $T$ для $g=1, p=3$

валентность данной вершины по отношению к ребрам самого редуцированного граффа. Пример такого факторизованного дерева $T$ для $p=3$ и $g=1$ представлен на рис. 1.

ЗАмечАниЕ 2. Множество собственных замкнутых путей граффа $T$ имеет естественный аналог в непрерывной проективной геометрии - это множество замкнутых геодезических линий (открытой) римановой поверхности.

Определим теперь для каждой точки $y \in T$ ее расстояние до редуцированного графа $d(y, F)$ по формуле $\inf _{x \in F} d(x, y)$ (иногда это расстояние будет сокрашенно записьваться как $d(y))$. Минимум достигается, если взять точку $y$ и единственньм образом определенную точку $x \in F$, которая называется образом $t(y) \in F$ точки $y$. Таким образом, каждая ветвь $B$ естественным образом может быть спроецирована на свою вершину (в другой терминологии корень, или образ) $t(y) \in F$.

Заметим, что терминология статьи [24], где теория рассеяния на графах исследовалась с точки зрения шрёдингеровских потенциалов, несколько отличается от принятой в данной статье. Так, редуцированный граф - это остов, базисньй граф в обозначениях Новикова. Далее, вместо бесконечных ветвей, растущих из вершин подграфа $F$, в этих работах рассматривались полубесконечные хвосты, растушие из соответствующих вершин (гнезд). Тем не менее, это описание очень близко описанию, используемому в данной статье, поскольку здесь рассматриваются (автоморфные) функции, зависяшие только от расстояния до редуцированного граффа; в этом подходе вершины хвоста как раз маркируют это расстояние, и оба подхода становятся эквивалентными в этом отношении. ${ }^{2}$

\section{2. Функции, операторы и потенциалы на графах}

2.1. Автоморфные функции и потенциалы на $T$. В первоначальном подходе Басса [11] фиксировалось унитарное представление $\rho: \pi_{1}(T) \rightarrow G L\left(v_{\rho}\right)$ групшы $Г$, и каждой вершине (и, в принципе, каждому ориентированныму ребру) дерева $D$ ставилось в соответствие линейное пространство $v_{\rho}$. Можно рассмотреть пространство $L\left(\chi, D, v_{\rho}\right)$ автоморфных функций на дереве $D$. Это функции $G(x)$, заданные на $V(D)$ (или на $\vec{L}(D))$, принимающие значения в представлении $v_{\rho}$ и удовлетворяющие условию $G(\gamma x)=\chi(\gamma) G(x)$ для всех $\gamma \in \Gamma$ и $x \in D$. Здесь $\chi(\gamma)$ - это левоинвариантный

\footnotetext{
${ }^{2}$ Важное различие между этими двумя подходами состоит в том, что в них исследуются различные типы операторов потенциала.
} 
характер группы $\Gamma$. Если $Y \subset D$ есть фундаментальная область группы $\Gamma$ в дереве $D$, то каждая функция $G \in L\left(\chi, D, v_{\rho}\right)$ полностью определяется своими значениями на $Y$.

В данной статье дается эквивалентное описание, которое близко по духу к широко используемым в физике решеточным калибровочным теориям. Именно, вместо того, чтобы рассматривать автоморфные функции, будут рассматриваться периодические функции на $D(F)$, которые поднимаются на граф $T$ (факторизованное дерево), в то время как множество нетривиальных характеров группы Г задается введением (унитарных) потенциалов на ребрах графа $T$.

Пусть $\mathscr{A}$ есть группа (или алгебра), а $v_{\rho}$ - конечномерное представление $\mathscr{A}$.

В работе [13] в основном рассматривался случай тривиального представления $\chi(\gamma) \equiv 1$; тем не менее, уже в этом простейшем примере проявились все основные свойства общей теории. В данной работе аналогичньй метод применяется для исследования случая произвольного унитарного (в общем случае неабелева) представления $\left(v_{\rho}, \chi\right)$.

ОПрЕДЕЛЕниЕ 4 . Пусть заданы группа $\mathscr{A}$ и ее конечномерное представление $v_{\rho}$. Каждой вершине и каждому ориентированному ребру графа $T$ поставим в соответствие пространство представления $v$ группы $\mathscr{A}$. Тогда соответствующие линейные пространства функиий, заданных в вершины или на ребрах графа и принимающих значения в $v_{\rho}$, обозначаются соответственно как $C_{0}=v^{|V|}$ и $C_{1}=v^{|\vec{E}|}$ (степень понимается как степень тензорного произведения).

В дальнейшем величины с тильдами понимаются как величины, заданные на редуцированном графе или на его универсальном накрытии. Например, линейные пространства $\widetilde{C}_{0}$ и $\widetilde{C}_{1}$ соответствуют именно вершинам и ребрам редуцированного графа $F$. Граф всегда предполагается замкнутым как симплициальньй комплекс, т.е. каждое ребро входит в этот граф вместе с обоими своими концами (вершинами).

Определим естественные билинейные формы на $C_{0}$ и $C_{1}$ : для $f, g \in C_{0}$ получим

$$
\langle f, g\rangle=\sum_{x \in V} f(x)^{*} g(x), \quad f(x), g(x) \in v_{\rho},
$$

и аналогично для $f, g \in C_{1}$.

ОПРЕДЕЛЕНИЕ 5. 1) Первый тип потенциала - это унитарные потенциаль на ребрах, $U_{\vec{e}} \in \mathscr{A}$, где $\mathscr{A}$ есть (неабелева) группа, а условие унитарности имеет вид $U_{\vec{e}}=U_{\overleftarrow{e}}^{-1}$. Это именно те потенциалы, которые возникают в формулировке Х. Басса [11].

2) Второй тип потенциалов-это эрмитовы потенииаль $A_{\vec{e}}$ на ребрах, лежащие в алгебре $\mathscr{A}$ и обладаюшие свойством $A_{\vec{e}}=A_{\overleftarrow{e}}=A_{\vec{e}}^{+}$(условие вешественности) [24].

3) Третий тип потенциалов, называемых потенциалами с нетривиальным кручением, - это потенциалы $U_{\vec{\mu}} \vec{\nu}$, определяемые парами последовательных ребер $(\vec{\mu}$ предшествует $\vec{\nu}$ ), такие что

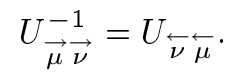


Еше одно естественное требование, часто накладьваемое на множество матриц кручения $U_{\vec{\mu}} \vec{\nu}$, имеет следующий вид. Пусть $\mu_{i}, i=1, \ldots, n,-$ ребра, входящие (в заданном циклическом порядке) в вершину граффа. Тогда для всех $1 \leqslant i, j, k \leqslant n$ вьполнено условие:

$$
U_{\vec{\mu}_{i} \overleftarrow{\mu}_{k}} U_{\vec{\mu}_{k} \overleftarrow{\mu}_{j}}=U_{\vec{\mu}_{i} \overleftarrow{\mu}_{j}}
$$

эти соотношения должны выполняться в каждой вершине и из них в частности следует, что $U_{\vec{e}_{i} \overleftarrow{e}_{i}}=I_{1}$ для каждого ребра $\vec{e} \in \vec{L}(D)$

2.2. Операторы Гекке на графах. В этом разделе $C_{0}$ и $C_{1}-$ соответствующие пространства функций на произвольном графе.

Рассмотрим сначала операторы, действующие внутри линейных пространств $C_{0}$ и $C_{1}$. Предполагается, что все эти операторы действуют на соответствующих универсальных накрывающих $D$ (или $D_{F}$ ) с естественным отождествлением (ориентированных) ребер и вершин $D$ с их прообразами в $T$ (или в $F$ ). Обозначим через $I_{0}$ и $I_{1}$ единичные операторы соответствуюших пространств $C_{0}$ и $C_{1}$.

1. Введем набор так называемых операторов Гекке $M_{n}: C_{0} \rightarrow C_{0}, n=1, \ldots, \infty$, таких, что их действие на базисных векторах имеет вид:

$$
M_{n} v_{x}=\sum_{y:\left|P_{x, y}\right|=n} U_{\vec{\mu}_{1}} \cdots U_{\vec{\mu}_{n}} v_{y}
$$

где произведение идет по всем ориентированным ребрам, входяшим в путь $P_{x, y}$ для $x, y \in D$.

Для тильда-операторов, относяшихся к симплициальным комплексам, связанным с редуцированным графом $F$, соответствующая определению (2.4) формула имеет вид:

$$
\widetilde{M}_{n} v_{x} \equiv \sum_{\substack{y:\left|P_{x, y}\right|=n \\ P_{x, y} \subset D_{F}}} U_{\vec{\mu}_{1}} \cdots U_{\vec{\mu}_{n}} v_{y}
$$

Понимается, что действие операторов (2.4) и (2.5) (так же, как и всех прочих операторов) продолжается по линейности на все пространство $C_{0}$.

2. Следуюший оператор - это оператор $Q: C_{0} \rightarrow C_{0}$, считаюший валентности в каждой вершине:

$$
Q v_{x}=(\# \text { соседей }-1) v_{x}
$$

Для однородного дерева оператор $Q$ пропорционален единичному с коэффициентом пропорциональности $p$. Однако, для неоднородного дерева, например, для $D_{F}$ оператор $Q$ обладает нетривиальными свойствами.

3. Определим $\Delta$-операторы $\Delta(u): C_{0} \rightarrow C_{0}$ и $\widetilde{\Delta}(u): \widetilde{C}_{0} \rightarrow \widetilde{C}_{0}$ :

$$
\Delta(u)=I_{0}-u M_{1}+u^{2} Q, \quad \widetilde{\Delta}(u)=I_{0}-u \widetilde{M}_{1}+u^{2} Q, \quad u \in \mathbb{C},
$$


которые имеют обратные (при $|u|<1 / p$ для тривиального характера) и играют важную роль в конструкции Басса. Иногда будет явно указьваться зависимость от представления, т.е. будет записьваться $\Delta_{\rho}(u)$.

Операторы $M_{n}(2.4)$ образую т базис в центре алгебры эндоморфизмов. Их алгебра умножения в дереве $D$ имеет вид:

$$
\begin{aligned}
& M_{1} M_{n}=M_{n+1}+p M_{n-1}, \quad n \geqslant 2, \\
& M_{1} M_{1}=M_{2}+(p+1) M_{0}, \quad M_{0} \equiv I_{0} .
\end{aligned}
$$

Если $\psi$ - собственньй вектор $M_{1}$ с собственным значением $t$, т.е.

$$
M_{1} \psi=t \psi
$$

то он также собственньй вектор для всех $M_{n}$ и

$$
M_{n} \psi=S_{n}(p, t) \psi
$$

где $S_{n}(p, t)$ - система ортогональных многочленов от $t$ с производяшим соотношением $t S_{n}(p, t)=S_{n+1}(p, t)+p S_{n-1}(p, t), S_{1}=t, S_{2}=t^{2}+p+1$.

В обшем случае неоднородного дерева $D_{F}$ оператор $Q$ очевидно некоммутативен с операторами Гекке, которые в свою очередь также перестают коммутировать между собой. Тем не менее, можно выписать алгебраические соотношения, аналогичные соотношениям (2.8), с использованием оператора $Q$ :

$$
\begin{aligned}
\widetilde{M}_{1} \widetilde{M}_{n} & =\widetilde{M}_{n+1}+Q \widetilde{M}_{n-1}, \quad n \geqslant 2, \\
\widetilde{M}_{1} \widetilde{M}_{1} & =\widetilde{M}_{2}+(Q+1) \widetilde{M}_{0}, \quad \widetilde{M}_{0}=\mathrm{id} .
\end{aligned}
$$

Более того,

$$
\sum_{n=1}^{\infty} u^{n} \widetilde{M}_{n} \widetilde{\Delta}(u)=\left(1-u^{2}\right) I_{0} .
$$

Из соотношения (2.12) следует, что оператор $\widetilde{\Delta}(u)$ есть производящая функиия для операторов $\widetilde{M}_{n}$.

4. Лапласиан унитарной теории - это оператор $\Delta(1)$. Для того, чтобы этот лапласиан был эрмитовьм оператором по отношению к скалярному произведению (2.1), на потенциал необходимо наложить дополнительное условие унитарности:

$$
U_{\vec{\mu}}^{+}=U_{\vec{\mu}}^{-1}
$$

В работе [24] лапласиан был определен как оператор типа оператора Шрёдингера, т.е. имел вид (2.7) с первым оператором Гекке $M_{1}$, заданным формулой $(2.4)$, в которой, однако, на ребрах графа унитарный потенциал $U_{\vec{\mu}}$ (групповой элемент) был 
заменен на эрмитов потенциал $A_{\vec{\mu}} \equiv A_{\overleftarrow{\mu}}$ (элемент алгебры). При этом соответствующий лапласиан

$$
\Delta v_{x}=\sum_{y:\left|P_{x, y}\right|=1}\left(A_{\vec{\mu}_{x y}} v_{y}-v_{x}\right)
$$

остается эрмитовым, но в общем случае никакого соотношения типа (2.11) не сушествует.

5. На пространстве $C_{1}$ сначала зададим отображение инверсии $J: C_{1} \rightarrow C_{1}$, которое меняет ориентации ребер на противоположные:

$$
\vec{J}=U_{\vec{e}} \overleftarrow{e} .
$$

Далее, в пространстве $C_{1}$ также существует набор операторов Гекке. Все они порождаются единственным оператором $T \equiv T_{1}: C_{1} \rightarrow C_{1}$.

ОПРЕДЕЛЕнИЕ 6. Первый оператор Гекке, действуюший на пространстве $C_{1}$, имеет вид:

$$
T \vec{e}=\sum_{\vec{e}_{\mu} \text { следует за } \vec{e}} A_{\vec{e}} \vec{e}_{\mu} \text {. }
$$

Очевидно, что теперь можно определить $T_{n}$ как сумму по всем последним ребрам ориентированных редуцированных путей длины $n+1$, начинающихся с данного ребра:

$$
T^{n}\left(\vec{e}_{0}\right)=\sum_{\left(\vec{e}_{0}, \vec{e}_{1}, \ldots, \vec{e}_{n}\right)_{\mathrm{red}}} \vec{e}_{n}
$$

Однако, в отличие от пространства $C_{0}$, соотношение меж ду операторами $T_{n}$ и $T_{1}$ выглядит совсем просто: $T_{n}=T_{1}^{n}$ для любого графа, и семейство этих операторов оказьвается коммутативным на любом дереве - однородном или неоднородном. Таким образом, для любого графа соответствуюшая производящая функция есть $I_{1}-u T_{1}$ :

$$
\sum_{n=0}^{\infty} u^{n} T_{n}\left(I_{1}-u T_{1}\right)=I_{1}, \quad u \in \mathbb{C} .
$$

Определение 6 легко обобщается на другие типы потенциалов из определения 5. Если заменить $U_{\vec{\mu}}$ на эрмитовы матрицы $A_{\vec{\mu}}$, то получается новиковский [24] потенциал на ребрах. Важные практические приложения возникают в случае потенциалов третьего типа (с нетривиальным кручением). Таким образом, в наиболее общем виде оператор $T$ задается следуюшей формулой:

$$
T \vec{e}=\sum_{\vec{e}_{\mu} \text { следует за } \vec{e}} U_{\vec{e}} F_{\vec{e}} \vec{e}_{\mu} \vec{e}_{\mu},
$$

где ни $U_{\vec{e}}$, ни $F_{\vec{e}} \vec{e}_{\mu}$ не обязаны удовлетворять условиям унитарности или эрмитовости; в формуле (2.19) потенциалы на ребрах, взятых с противоположными ориентациями, могут быть вообше не связаны между собой. Тем не менее, результаты следуюшего раздела, выражаюшие $L$-функцию через оператор $T$, остаются справедливыми.

Отметим два важных примера потенциалов (2.19). Первьй (см. раздел 6) - это описание в терминах графов [19], [20] пространства Тейхмюллера комплексных римановых поверхностей; второй известньй пример - это модель Изинга на решетке, статистическая сумма которой задается детерминантом оператора Каца-Уорда [25], [26]. 
2.3. Операторы смешивания между пространствами $C_{0}$ и $C_{1}$. Следуя работе [11], введем набор операторов, действуюших между пространствами $C_{0}$ и $C_{1}$.

1. Зададим, во-первых, два граничных отображсения $\partial_{1}, \partial_{0}: C_{1} \rightarrow C_{0}$ таких, что

$$
\partial_{1} \vec{e}=A_{\vec{e}} x_{1}
$$

и

$$
\partial_{0} \vec{e}=x_{0}
$$

2. Введем также кограничньй оператор $\sigma_{0}: C_{0} \rightarrow C_{1}$ :

$$
\sigma_{0} x=\sum_{y:\left|P_{x, y}\right|=1} \vec{e}_{(x, y)},
$$

которьй сопоставляет вершине $x$ линейную комбинацию всеx ребер, исходящих из этой вершины. (В работе [11] был также определен оператор $\sigma_{1}$, которьй сопоставляет вершине $x$ линейную комбинацию всех входящих ребер. Однако это определение, имеюшее хороший смысл в случае тривиального потенциала, становится неестественным, как только вводится неабелев потенциал. В дальнейшем, как будет видно, вся конструкция может быть построена без использования оператора $\sigma_{1}$.)

2.4. Соотношения между операторами. Определим полные (половинные) размерности пространств $C_{0}$ и $C_{1}$ :

$$
r_{0}=\operatorname{rank}\left(C_{0}\right), \quad r_{1}=\operatorname{rank}\left(C_{1}\right) / 2,
$$

и рассмотрим вспомогательные операторы

$$
\partial(u)=\partial_{0} u-\partial_{1} ; \quad \sigma(u)=\sigma_{0} u
$$

Стандартньй граничный оператор имеет при этом вид: $\partial \equiv \partial_{0}-\partial_{1}=\partial(1)$.

Путем прямых вычислений можно легко показать, что указанные операторы удовлетворяют следующим соотношениям [11], выполненным для произвольного графа:

$$
\begin{gathered}
\partial_{0} \sigma_{0}=Q+1 ; \quad \partial_{1} \sigma_{0}=M_{1} ; \quad T=\sigma_{0} \partial_{1}-J ; \\
\partial(u) \sigma(u)=\Delta(u)-\left(1-u^{2}\right) I_{0} ; \\
\sigma(u) \partial(u)=u(T+J)\left(u J-I_{1}\right) .
\end{gathered}
$$

Важное утверждение, доказанное Бассом, устанавливает связь между спектральными задачами для оператора Лапласа и для оператора Гекке $T_{1}$.

ЛЕмма 1 [11]. В теориях с потенииалами первого типа из определения 2.2 выполнено соотночение:

$$
\operatorname{det}_{C_{1}}\left(I_{1}-u T_{1}\right)=\left(1-u^{2}\right)^{r_{0}-r_{1}} \operatorname{det}_{C_{0}} \Delta(u) .
$$


ДокАЗАТЕЛьСтво. Введем еще две вспомогательные матришы, действуюшие на прямой сумме пространств $C_{0} \oplus C_{1}$ :

$$
L=\left[\begin{array}{cc}
\left(1-u^{2}\right) I_{0} & \partial(u) \\
0 & I_{1}
\end{array}\right], \quad M=\left[\begin{array}{cc}
I_{0} & -\partial(u) \\
\sigma(u) & \left(1-u^{2}\right) I_{1}
\end{array}\right] .
$$

Тогда из соотношений (2.25) следует, что

$$
L M=\left[\begin{array}{cc}
\Delta(u) & 0 \\
\sigma(u) & \left(1-u^{2}\right) I_{1}
\end{array}\right] \text { и } M L=\left[\begin{array}{cc}
\left(1-u^{2}\right) I_{0} & 0 \\
\sigma(u)\left(1-u^{2}\right) & \left(I_{1}-u T\right)\left(I_{1}-u J\right)
\end{array}\right] .
$$

У тверждение леммы 1 получается, если приравнять следы матриц $L M$ и $M L$; единственное вычисление, которое при этом необходимо проделать, - это нахождение детерминанта оператора $I_{1}-u J$. Этот оператор блочно-диагонален в базисе (неориентированных) ребер. Поскольку каждое ребро допускает две ориентации, то получим, что

$$
\operatorname{det}\left(I_{1}-u J\right)=\operatorname{det}\left[\begin{array}{cc}
I_{1} & -u U \\
-u U^{-1} & I_{1}
\end{array}\right]^{|\vec{E} / 2|}=\left(1-u^{2}\right)^{r_{1}}
$$

для любой матрицы $U$. Заметим, что $r_{1}$ - это в точности размерность представления группы $U$, умноженная на $|E|$, где $|E|$ - полное число неориентированных ребер. Таким образом, $r_{0}-r_{1}=\operatorname{dim} U \times(|V|-|E|)$, где разность между числом вершины и числом (неориентированных) ребер есть в точности $1-g$ - эйлерова характеристика граф̆а.

В случае потенциалов общего положения второго и третьего типа из определения 5 соотношения типа утверждения леммы 2 теряют справедливость. Тем самым, спектральные задачи операторов $\Delta(u)$ и $T(u)$ становятся несвязанньми. Тем не менее, в случае графов с потенциалами третьего типа из определения 5 и с наложенными дополнительньми условиями (2.2) и (2.3) эти две спектральные задачи можно связать, если видоизменить исходный граф̆ путем "раздутия" его вершин. Эта процедура явно описана в разделе 6 .

\section{3. $L$-функция}

ОПРЕДЕЛЕНИЕ 7 [11]. Пусть $v_{\rho}$ есть $\mathbb{C}^{k}$-модуль с характером $\chi_{\rho}: T(C[T]) \rightarrow \mathbb{C}^{k}$. Тогда определим $L$-функиию Ихарь-Сельберга $L(\rho, u)$ :

$$
L(\rho, u)=\prod_{\{\varpi\} \in \Gamma} \operatorname{det}\left(I_{v}-u^{l(\varpi)} \rho(\varpi)\right)^{-1},
$$

где произведение берется по классам сопряженности всех примитивных элементов группы $Г$ или, эквивалентно, по всем собственным ориентированным замкнутым путям в графе $T$, не содержашим подпериодов, $l_{\varepsilon}$ суть длины этих путей, а произведение $\rho(\varpi)=U_{\vec{e}_{1}} \cdots U_{\vec{e}_{l_{\varepsilon}}}$ элементов групы $\mathscr{A}$ берется по пути, соответствуюшему элементу $\varpi \in \Gamma$.

Основная теорема К.-И. Хашимото [9], [10] была удобным образом сформулирована и доказана X. Бассом [11]. 
Теорема 1. Пусть $\rho: \Gamma \rightarrow G L(V)$ - конечномерное комплексное представление группь Г. Тогда L-функиия (3.1) есть рациональная функиия переменных и:

$$
L(\rho, u)^{-1}=\operatorname{det}_{C_{1}}\left(I_{1}-u T_{1}\right)
$$

əде $T_{1}$ - оператор (2.16).

Следующее утверждение вытекает из леммы 1 и теоремы 1.

СЛЕДСТВИЕ 1. В предположсении выполнения условия унитарности (2.13) $L$-функция может бить представлена через детерминант оператора $\Delta_{\rho}(u)$ :

$$
L(\rho, u)^{-1}=\left(1-u^{2}\right)^{r_{0}-r_{1}} \operatorname{det}_{C_{0}}\left(\Delta_{\rho}(u)\right)
$$

Произведение в $(3.1)$ абсолютно сходится в области $\left\{u:|u|<\left(q_{\max }|\mathscr{A}|\right)^{-1}\right\}$, где $q_{\text {max }} \leqslant p$ - максимальное число инцидентности в дереве $D_{F}$ и $|\mathscr{A}|$ - модуль максимального элемента $U_{i j}^{\mu}$. Для логарифмической производной $L$-функции $L(\rho, u)$ вьполняется следуюшее соотношение:

$$
\frac{d}{d u} \log (L(\rho, u))=\sum_{\{\gamma\}} \Lambda(\gamma) \operatorname{tr}(\rho(\gamma)) u^{l(\gamma)-1}
$$

где сумма берется по всем классам сопряженности $\{\gamma\}$, отличным от $\{1\}$.

ДокАЗАТЕЛЬСТво теоРемы 1. Выберем в дереве $D$ некоторую фундаментальную область $\mathscr{D}(\Gamma)$ групшы симметрий Г. Вычислим вкладнекоторого ребра $\vec{e}_{1} \in \mathscr{D}(\Gamma)$ в след оператора $T^{m}$. Ненулевой вклад в $\operatorname{tr} T^{m}$ дают лишь те $\vec{e}_{i} \in D$, которые

1 ) лежат на расстоянии $m$ от исходного ребра $\vec{e}_{1}$ (расстояние измеряется вдоль некоторого редуцированного пути $\left.\vec{e}_{1} \vec{e}_{2} \ldots \vec{e}_{m+1}\right)$;

2) ориентированы вдоль этого редуцированного пути.

Ключевой момент доказательства состоит в следующем наблюдении. Рассмотрим действие некоторого элемента $\gamma \in \Gamma$ на ориентированные ребра дерева $D$ (см. рис. 2). Легко видеть, что если ребро не принадлежит инвариантной оси $D(\gamma)$ этого элемента (подобно ребру $\vec{e}_{x}$ на рис. 2), то ориентация образа этого ребра при действии элемента $\gamma, \gamma \vec{e}_{x}$ с необходимостью противоположсна ориентации редуцированного пути, выходящего из ребра $\vec{e}_{x}$ и заканчиваюшегося на ребре $\gamma \vec{e}_{x}$. Таким образом, ни одно из таких ребер не дает вклада в след оператора! Только лишш те ребра, которые подобно $\vec{e}_{1}$ на рис. 2 принадлежат инвариантной оси $D(\gamma)$, сохраняют свои ориентации вдоль $D(\gamma)$ при действии элемента $\gamma$ (задающего перенос вдоль оси $D(\gamma)$ ). Например, для $\vec{e}_{1}$ получим $\vec{e}_{l+1}=\gamma \vec{e}_{1}$ (см. рис. 2$)$.

ЗАмЕчАниЕ. Это наблюдение объясняет, почему так удобно исследовать $\operatorname{tr} T^{m}$, а не, скажем, $M_{m}-m$-й оператор Гекке в пространстве $C_{0}$. Дело в том, что в $\operatorname{tr} M_{m}$ в принципе дают вклад все пары вершин $x, y \in D$, для которых $d(x, y)=m$ и $y=\gamma x$. Среди таких вершин встречаются как лежащие, так и не лежащие на инвариантной оси $D(\gamma)$. 


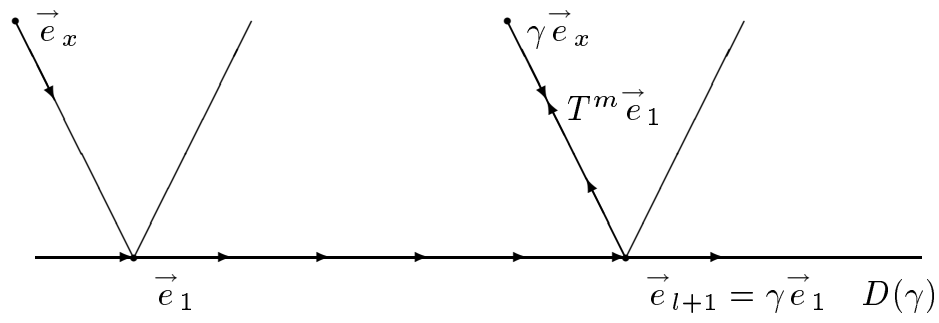

Рис. 2. Действие группового элемента $\gamma \in \Gamma$ с длиной $l(\gamma)=l$ на ребрах дерева $D$. Заметим, что $\gamma \vec{e}_{x} \neq T^{m+1} \vec{e}_{1}$ ни для какого $m$

Возвращаясь к пространству $C_{1}$, зафиксируем на некоторое время ребро $\vec{e}_{1} \in$ $D(\Gamma) \subseteq D$. Как отмечалось вьше, для примитивного класса сопряженности $\varpi$ может сушествовать более одной инвариантной оси $D(\varpi) \subset D_{F}$ такой, что $\vec{e}_{1} \in D(\varpi)$. Введем обозначения: пусть $\vec{a}_{i}$ обозначают ориентированные ребра самого редуцированного граффа $F$, и тем самым каждое ребро $\vec{e}_{i} \in D_{F}$ есть образ ребра $\vec{a}_{i} \in F$ с естественным образом сохраненной ориентацией. Поставим в соответствие каждому элементу $\gamma \in \Gamma$ периодическую последовательность "букв" (циклическое слово)

$$
D(\gamma) \simeq \ldots\left(\vec{a}_{1} \vec{a}_{2} \ldots \vec{a}_{l}\right)\left(\vec{a}_{1} \vec{a}_{2} \ldots \vec{a}_{l}\right) \ldots
$$

где $l \equiv \Lambda(\gamma)=l(\varpi)$ - длина производящего элемента стабилизирующей подгруппы элемента $\gamma$.

Отметим, что элементы Г задают символьную динамику “слов" (слова Линдона, см. [37], [38]), составленных из "букв" $\vec{a}_{i}$, которыемогут быть некоммутативными элементами групшы $\mathscr{A}$. В этом случае не обязано вьполняться даже условие унитарности (2.13); утверждение теоремы 1 остается справедливым, даже если символы (буквы) $\vec{a}_{i}$ и $\overleftarrow{a}_{i}$ вообще никак не связаны между собой. Таким образом, до конща доказательства буквы $\vec{a}_{i}$ будут просто отождествлены с элементами $U_{\vec{e}_{i}} \cdot \vec{e}_{i}$, а доказательство остается справедливым для любой (в том числе неабелевой) группы или алгебры, элементы которой заданы на ориентированных ребрах графа $F$.

Ясно, что если $l(\gamma)=m$, то $l(\varpi)=l, l \mid m$, т.е. $l$ есть делитель числа $m$. Учтем, что в циклическом слове $\vec{a}_{1} \vec{a}_{2} \ldots \vec{a}_{l}\left(\vec{a}_{l+1} \equiv \vec{a}_{1}\right)$ могут встречаться повторные буквы $\vec{a}_{i}$. Более того, эта последовательность может содержать подпериоды в случае, когда $\gamma$ не является примитивным элементом, однако очевидно, что минимальная длина $l$ такого подпериода обязана быть делителем $m, l \mid m$.

Зафиксируем теперь элемент $\vec{a}_{1}-$ прообраз $\vec{e}_{1}$. Чтобы найти его вклад в $\operatorname{tr} T^{m}$, необходимо найти все различные циклические выражения

$$
\vec{a}_{1} \vec{a}_{2} \ldots \vec{a}_{m}\left(\vec{a}_{m+1}=\vec{a}_{1}\right)
$$

содержашие это элемент. Установим теперь связь между множествами (3.5) и (3.6). 
1. Поставим в соответствие каждой конечной циклической последовательности $\vec{a}_{1} \ldots \vec{a}_{m}\left(\vec{a}_{1}\right)$ однозначно определенную бесконечную периодическую последовательность $\ldots\left(\vec{a}_{1} \ldots \vec{a}_{m}\right)\left(\vec{a}_{1} \ldots \vec{a}_{m}\right) \ldots$, отвечающую однозначно определенному примитивному классу сопряженности $\{\varpi\}$, длина которого $l(\varpi)=l \mid m$.

2. Наоборот, выберем элемент из $\{\varpi\}$ или, эквивалентно, некоторую периодическую редуцированную последовательность

$$
\ldots\left(\vec{a}_{i_{1}} \vec{a}_{i_{2}} \ldots \vec{a}_{i_{l}}\right)\left(\vec{a}_{i_{1}} \vec{a}_{i_{2}} \ldots \vec{a}_{i_{l}}\right) \ldots
$$

не содержащую подпериодов и имеющую минимальный период $l=l(\varpi)$. Если (ориентированное) ребро $\vec{a}_{1}$ содержится $d_{1}$ раз среди ребер $\left\{\vec{a}_{i_{1}}, \ldots, \vec{a}_{i_{l}}\right\}$ и, более того, $l$ - делитель числа $m$, то имеются в точности $d_{1}$ различных последовательностей $\vec{a}_{1} \ldots \vec{a}_{m}\left(\vec{a}_{1}\right)(3.6)$, которые содержатся в (3.7). Наконец, производя суммирование по всем ребрам графа $F$ (или фундаментальной области $\mathscr{D}(\Gamma) \in D$ ), т.е. вычисляя след оператора $T^{m}$, найдем, что вклад, который последовательность (3.7) дает в этот след, имеет вид:

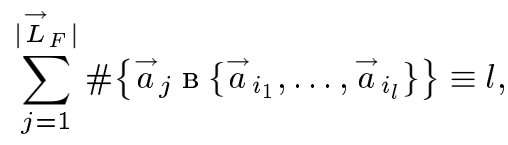

где $l$ - полная длина примитивного элемента $\varpi$. Таким образом, получим следующую замечательную формулу:

$$
\operatorname{tr} T^{m}=\sum_{l \mid m} l \cdot \sum_{\{\varpi: l(\varpi)=l\}} \operatorname{tr}\left[\rho(\varpi)^{m / l}\right]
$$

где сумма берется по всем примитивным классам сопряженности группы Г.

Теперь из формулы (3.4) следует представление для $L$-функции:

$$
\begin{aligned}
u \frac{d}{d u} \log (L(\rho, u)) & =\sum_{\{\varpi\}} \sum_{n=1}^{\infty} l(\varpi) \cdot u^{n l(\varpi)} \operatorname{tr}\left[\rho(\varpi)^{n}\right] \\
& \stackrel{(3.9)}{=} \sum_{k=1}^{\infty} \operatorname{tr} T^{k} \cdot u^{k}=-u \frac{d}{d u} \log \operatorname{det}(1-u \cdot T) .
\end{aligned}
$$

Это завершает доказательство теоремы.

\section{4. Спектральная задача на бесконечных графах}

Спектральная задача состоит в исследовании таких функций $\psi \in C_{0}$, что

$$
L \psi=\lambda \psi, \quad \lambda \in \mathbb{C},
$$

где лапласиан $L$ имеет вид:

$$
L=M_{1}-(Q+1) .
$$


Определим сначала решение задачи (4.1) на ветвях (хвостах). Как и раньше, будем предполагать граф $T$ однородным $(p+1)$-валентным и бесконечным. Рассмотрим ветвь (хвост) графа $T$ с началом в вершине $x_{0}$ (нижний индекс $i$ в последуюших вычислениях соответствует расстоянию до вершины $\left.x_{0}\right)$. Обозначив $\psi\left(x_{i}^{(s)}\right)=v_{i}^{(s)}$, получим уравнение:

$$
\sum_{s=1}^{p} v_{i+1}^{(s)}+v_{i-1}^{(\cdot)}-(p+1) v_{i}=\lambda v_{i}, \quad i>0
$$

где $x_{i-1}^{(\cdot)}$ - однозначно определенная вершина (из $(i-1)$-го слоя), которая предшествует $x_{i}$ (при движении вдоль ветви по направлению из корня).

Общее решение уравнения (4.3) имеет вид:

$$
v_{i}^{(s)}=\sum_{ \pm} \alpha_{ \pm}^{i}\left(A_{i-1}^{\left(s_{i-1}\right)}\right)^{-1} \cdots\left(A_{1}^{\left(s_{1}\right)}\right)^{-1} v_{ \pm}^{0},
$$

где произведение берется по всем ребрам, входящим в путь $P_{x_{0}, x_{i}^{\left(s_{i}\right)}}, v_{+}^{0}$ и $v_{-}^{0}-$ постоянные векторы из $V$, а $\alpha_{ \pm}$- решения уравнения

$$
p \alpha_{ \pm}^{2}-(p+1+\lambda) \alpha_{ \pm}+1=0 ; \quad t \equiv \frac{p+1+\lambda}{2 p}, \quad \alpha_{ \pm}=t \pm \sqrt{t^{2}-1 / p} .
$$

Векторы

$$
\left[v_{i \pm}^{(s)}\right]_{\rho} \equiv \alpha_{ \pm}^{i}\left(U_{i-1}^{\left(s_{i-1}\right)}\right)^{-1} \ldots\left(U_{1}^{\left(s_{1}\right)}\right)^{-1}\left(v_{ \pm}^{0}\right)_{\rho}
$$

где $\left(v_{ \pm}^{0}\right)_{\rho}-\rho$-я компонента вектора $v_{ \pm}^{0}$, остаются коллинеарньми при каждом $i$, и их отношение $\left(\alpha_{+} / \alpha_{-}\right)^{i}\left(v_{+}^{0}\right)_{\rho} /\left(v_{-}^{0}\right)_{\rho}$ зависит лишь от уровня $i$. Поэтому естественно связать данные $\left(v_{+}^{0}\right)_{\rho}$ и $\left(v_{-}^{0}\right)_{\rho}$ с данными матришы рассеяния на графе $T$. Для этого назовем решения (4.4), у которых только компоненты $v_{+}^{0}$ отличны от нуля, nадающей волной, а решения (4.4) с отличньми от нуля компонентами $v_{-}^{0}-$ отраженной волной.

Теперь становится понятным, почему можно связать описания в терминах ветвей и хвостов. В качестве предварительного шага можно с помошью калибровочного преобразования избавиться от зависимости от потенциалов на ребрах ветвей. Для этого достаточно положить

$$
v_{i}^{(s)} \rightarrow U_{1}^{\left(s_{1}\right)} U_{2}^{\left(s_{2}\right)} \ldots U_{i-1}^{\left(s_{i-1}\right)} v_{i}^{(s)} .
$$

Тогда в преобразованных переменных спектральная задача соответствует тривиальным потенциалам на ребрах и

$$
\left[v_{i \pm}\right]_{\rho}=\alpha_{ \pm}^{i}\left[v_{0 \pm}\right]_{\rho}
$$


для любого пути длины $i$, начинающегося в корне ветви. Поэтому в дальнейшем можно положить $U_{\vec{\mu}}=I_{v}$ на всех внешних (т.е. не входящих в минимальньй редуцированный подграф̆) ребрах.

После этого отождествим все вершины и ребра, отстояшие на одинаковое расстояние от корня ветви, положив

$$
v_{i}^{(s)} \rightarrow p^{(i-1) / 2} v_{i} \text { при } i>0,
$$

и опустим теперь уже ненужные верхние индексы $(s)$ (см. рис. 3 ). При этом получается в точности картина, возникаюшая в описании работы [24], где в каждой вершине остова (или в принятых здесь обозначениях редуцированного графа) начинается конечное (возможно нулевое) число хвостов (полубесконечных прямых). Переход (4.7) таков, что скалярное произведение (2.1) сохраняется. Одновременно, необходимо растянуть и сдвинуть соответствующее собственное значение: $\lambda \rightarrow\left[\lambda+(\sqrt{p}-1)^{2}\right] / \sqrt{p}$; только после этого ответы для двух ветвей решения (4.5) в данном подходе и в подходе [24] действительно совпадут.

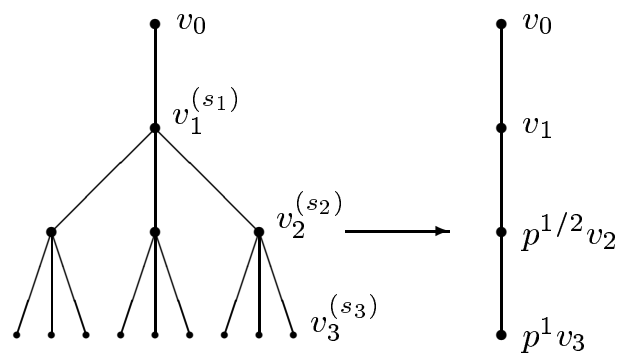

Рис. 3. Переход “ветвь $\rightarrow$ хвост” на внешних ребрах графа $T$

ОПРЕДЕЛЕНИЕ 8. Пусть граничные точки $x_{i} \in \partial F-$ это те точки редуцированного графа $F$, для которых число внешних хвостов, начинаюшихся в этих точках, omлично от нуля. Также удобно ввести естественные вложения линейных пространств $(\partial F)_{0,1} \subset \widetilde{C}_{0,1}$ (напомним, что $\left.(F)_{0,1} \equiv \widetilde{C}_{0,1}\right)$.

4.1. Сферические функции на факторизованных деревьях. Обобщим теперь понятие сферических функций на случай графа $T$. Сначала рассмотрим множество сферических функций на самом дереве $D$. Выберем некоторую вершину $x \in V(D)$, назовем ее иентром дерева. Тогда сферическая функиия $\psi(n, x)$ - это такой собственньй вектор оператора $M_{1}, M_{1} \psi(n, x)=t \psi(n, x)$, который зависит лиш от расстояния от точки $x$ по ребрам дерева, т.е. эта функция постоянна на всех сферах $S(n, x) \equiv\left\{y \in V(D):\left|P_{x, y}\right|=n\right\}$. В случае единичного потенщиала (тривиального представления групшы $\Gamma$ ) получим:

$$
\psi(n, x)=a_{+} \alpha_{+}^{n}-a_{-} \alpha_{-}^{n},
$$

где (ср. с (4.5))

$$
\alpha_{+} \alpha_{-}=1 / p
$$


и полагая $\psi(0, x)=1$, получим, что

$$
a_{+}=\frac{p \alpha_{+}-\alpha_{-}}{(p+1)\left(\alpha_{+}-\alpha_{-}\right)}, \quad a_{-}=\frac{p \alpha_{-}-\alpha_{+}}{(p+1)\left(\alpha_{+}-\alpha_{-}\right)} .
$$

Для того чтобы определить аналогичную функцию на обшем графе $T$, аналогом “центра" которого является редуцированньй граф $F$, рассмотрим суперпозииию решений (4.8) с источниками $s_{y}$, размешенными в вершинах поддерева $D_{F}$ :

$$
\psi(x)=\sum_{y \in D_{F}} s_{y} \psi(d(x, y), y)
$$

Это распределение источников должно быть таким, чтобы функция $s_{y}$ была периодической относительно действия группы $\Gamma: s_{\gamma y}=s_{y}$ для всех $\gamma \in \Gamma$ и $y \in D_{F}$. Поведение такого решения на каждой ветке, “растущей” из $\partial F$, описывается формулой (4.8), в которой предэкспоненциальные множители зависят только от точки множества $\partial F$, в которую проецируется эта ветвь.

Следующая полезная для описания функция - это ядерная функция $K$ :

$$
K(z, y \mid x)=\sum_{\gamma \in \Gamma} x^{d(z, \gamma(y))} \times \prod_{\vec{\mu}_{i} \in P_{z, \gamma(y)}} U_{\vec{\mu}_{i}},
$$

в которой $z, y$ - точки поддерева $D_{F}$. Эта функция, очевидно, периодична относительно действия группы Г по отдельности на каждый из ее аргументов. Поэтому она однозначно определена также и на самом графе $T$. В случае тривиального представления $\chi \equiv 1$ эта функция к тому же симметрична относительно перестановок $z$ и $y$. Функцию (4.10) теперь можно записать как

$$
\psi(x)=\sum_{y \in F} s_{y}\left[a_{+} K\left(t(x), y \mid \alpha_{+}\right) \alpha_{+}^{d(x)}-a_{-} K\left(t(x), y \mid \alpha_{-}\right) \alpha_{-}^{d(x)}\right]
$$

где $t(x) \in F$ - образ точки $x \in D$. (Если $x \in F$, то $t(x)=x$.) Как и ранњше, будем назьвать часть выражения (4.12), пропорциональную $\alpha_{+}^{d(x)}$, падающей волновой функцией и часть, пропорциональную $\alpha_{-}^{d(x)}$, - отражсенной волновой функцией. Таким образом, функция $\psi(x)$ может быть представлена в следуюшем общем виде:

$$
\psi(x)=A_{\mathrm{adv}}(t(x)) \alpha_{+}^{d(x)}-A_{\mathrm{ret}}(t(x)) \alpha_{-}^{d(x)} \equiv \psi_{+}(x)-\psi_{-}(x) .
$$

ПРЕДЛОЖЕНИЕ 1. По Каждому полному набору векторов $v_{+}^{0}\left(x_{i}\right), x_{i} \in \partial F$, набор векторов $v_{-}^{0}\left(x_{i}\right)$ определяется однозначно при всех $t \in \mathbb{C}$, кроме, возможсно, конечного числа точек (точек дискретного спектра).

Важньй случай функций (4.13) - тот, где $\psi_{+}(x)$ отлична от нуля только на одной из ветвей, растуших из одной, выделенной точки $\partial F$. Тогда можно определить матрищу рассеяния следующим образом. 
ОПрЕДЕЛЕниЕ 9. Матрица рассеяния ( $S$-матрица) $S(t)$ - это квадратная матрица размера $\operatorname{rank}(\partial F)_{0}$ с элементами $s_{i_{\alpha}, j_{\beta}},\left|\left\{i_{\alpha}\right\}\right|=\left|\left\{j_{\beta}\right\}\right|=\operatorname{rank}(\partial F)_{0}$, где $s_{i_{\alpha}, j_{\beta}}$ есть значение $\left(v_{-}^{0}\right)_{\beta}\left(x_{j}\right)$ при $\left(v_{+}^{0}\right)_{\gamma}\left(x_{k}\right) \equiv \delta_{\alpha \gamma} \delta_{k i}$, и $\left(v_{ \pm}^{0}\right)$ задают решение спектральной задачи (4.1) на полном графе, т.е. элементы $S$-матрицы отвечают решениям уравнения (4.1) вида

$$
\psi_{i_{\alpha}}^{+}-\sum_{\left\{j_{\beta}\right\}} s_{i_{\alpha}, j_{\beta}} \psi_{j_{\beta}}^{-}
$$

Отложим подробное исследование спектральных свойств $S$-матрицы до следующего раздела и сформулируем пока основную теорему, связьваюшую $S$-матрицу и детерминанты операторов, заданных на редуцированном графе.

Теорема 2. В случае унитарных потенциалов, заданных на ребрах графа, получим:

$$
\operatorname{det} S(t)=\left(\frac{\alpha_{+}}{\alpha_{-}}\right)^{\operatorname{rank}(F)_{0}} \frac{\operatorname{det}\left(\Delta_{\rho}\left(\alpha_{-}\right)\right)}{\operatorname{det}\left(\Delta_{\rho}\left(\alpha_{+}\right)\right)},
$$

где $\left(\Delta_{\rho}\left(\alpha_{+}\right)\right)$- оператор $(2.7)$, в котором явно указана зависимость от представления $\rho$.

ДокАЗАТЕльство. Каждое решение спектральной задачи (4.1), имеющее вид (4.4) вне редуцированногографа, может быть расшеплено на падающую и отраженную парциальные волновые функции в полном графе (см. (4.13)), где

$$
\psi_{ \pm}(x)=\sum_{x_{\alpha} \in F} \sum_{P_{x_{\alpha}, x}} a_{ \pm} \alpha_{ \pm}^{\left|P_{x_{\alpha}, x}\right|} \prod_{\vec{e}_{i} \in P_{x_{\alpha}, x}} U_{\vec{e}_{i}}^{-1} s\left(x_{\alpha}\right)
$$

Здесь $s\left(x_{\alpha}\right): F_{0} \rightarrow V$ - это функиия источников, а числа $a_{ \pm}$заданы формулой (4.9).

Заметим, что подобное "расщепление" на падающую и отраженную волны или, эквивалентно, задание функции источников $s\left(x_{\alpha}\right)$ определено однозначно всюду вовне $F$ (при этом расшепление на такие (парциальные) волны однозначно определено и в граничных точках $x \in \partial F$ ), но эта процедура становится неоднозначной во внутренних точках редуцированного графа $F$.

Для заданной собственной функции $\psi(x)$ получим, что в пространстве функций $s(x)$ (в действительности, это пространство есть пространство $\widetilde{C}_{0}$ ) существует линейное подпространство функций источников, генерирующих одну и ту же указанную волновую функцию. Например, если $x_{0} \in F, x_{0} \notin \partial F$, то можно найти по крайней мере две линейно независимых функции источников $s_{1}(x)=\left\{0, x \neq x_{0} ; 1, x=x_{0}\right\}$ и $s_{2}(x)=\left\{0, d\left(x, x_{0}\right) \neq 1 ; 2 p / t, d\left(x, x_{0}\right)=1\right\}$, задающих одну и ту же собственную функцию $\psi(x)$. Более того, при почти всех $\lambda$ и $s(x) \in \widetilde{C}_{0}$ можно найти единственную функцию источников $\bar{s}(x) \in \widetilde{C}_{0}$ такую, что обе функции $s(x)$ и $\bar{s}(x)$ порождают одну и ту же собственную функцию $\psi(x)$ и при этом $\bar{s}(x) \equiv 0$ во всех внутренних точках $F$.

Таким образом, проблема состоит в том, чтобы задать каким-либо образом "удобное" расшепление (4.13) (аналог конечномерной “фиксации калибровки" в теории поля). 
Рассмотрим собственные значения $c_{n}$ матрицы $S(t)$. Это числа, для которых сушествуют такие решения задачи (4.1), при которых переменные $\left(v_{+}^{0}\right)_{\alpha}\left(x_{i}\right)$ и $\left(v_{-}^{0}\right)_{\alpha}\left(x_{i}\right)$ могут быть выбраны пропорииональными друг другу:

$$
\left(v_{+}^{0}\right)_{\alpha}\left(x_{i}\right)=c_{n}\left(v_{-}^{0}\right)_{\alpha}\left(x_{i}\right) \text { для всех } \alpha \text { и } x_{i} \in \partial F .
$$

Основной момент конструкции состоит в распространении условия (4.16) на все (внешние и внутренние) вершины редуцированного граффа $F$.

ОПРеДЕЛЕниЕ 10. Пусть сферическая функиия на графе $T$ - это решение спектральной задачи (4.1), имеющее форму (4.15) и такое, что выполнено следующее условие:

$$
\left[\psi_{+}(x)\right]_{\alpha} /\left[\psi_{-}(x)\right]_{\alpha}=c_{n}\left(\frac{\alpha_{+}}{\alpha_{-}}\right)^{\operatorname{dist}(x, F)},
$$

где $c_{n}$ - константа, не зависяшая ни от точки $x$, ни от индекса представления $\alpha$, и $\operatorname{dist}(x, F)$ - хорошо определенное расстояние между точкой $x$ и редуцированным графом $F$ (для $x \in F, \operatorname{dist}(x, F)=0$ ).

Лemma 2.

$$
\operatorname{det} S(t)=\prod_{n} c_{n}
$$

В действительности, условие (4.17) фиксирует выбор допустимой функции распределения источников $s\left(x_{\alpha}\right)$. В то же время, это условие задает систему линейных однородных уравнений, которая допускает ненулевое решение только для конечного числа $c_{n}$. Ниже эта система будет выписана через операторы Гекке (2.5), действуюшие на графе $F$.

Рассматривая уравнение (4.17) в вершинах редушированного графа, получим:

$$
\sum_{x_{\alpha} \in F} a_{+} \alpha_{+}^{\left|P_{x_{\alpha}, x}\right|}\left(\prod_{\vec{e}_{i} \in P_{x_{\alpha}, x}} U_{\vec{e}_{i}}^{-1}\right) s\left(x_{\alpha}\right)=c_{n} \sum_{x_{\alpha} \in F} a_{-} \alpha_{-}^{\left|P_{x_{\alpha}, x}\right|}\left(\prod_{\vec{e}_{i} \in P_{x_{\alpha}, x}} U_{\vec{e}_{i}}^{-1}\right) s\left(x_{\alpha}\right),
$$

или

$$
\sum_{y \in F} s_{y} a_{+} K\left(z, y \mid \alpha_{+}\right)=c_{n} \sum_{y \in F} s_{y} a_{-} K\left(z, y \mid \alpha_{-}\right) .
$$

Тогда очевидно, что

$$
\prod_{n} c_{n}=\frac{\operatorname{det} K\left(x, y \mid \alpha_{+}\right)}{\operatorname{det} K\left(x, y \mid \alpha_{-}\right)}\left(\frac{a_{+}}{a_{-}}\right)^{\operatorname{rank}(F)_{0}}
$$

где $K(x, y \mid \alpha)$ - ядро линейного оператора, действуюшего на пространстве $\widetilde{C}_{0}$ (заметим, что так как подграф $F$ содержит все петли графа $T$ и связен, то для любых $x, y \in F$ любой путь $P_{x, y}$, связывающий эти вершины, лежит целиком в $\left.F\right)$ :

$$
(K s)(x)=\sum_{y \in F} \sum_{P_{x, y}} \alpha^{\left|P_{x, y}\right|}\left(\prod_{\vec{e}_{i} \in P_{x, y}} U_{\vec{e}_{i}}^{-1}\right) s(y) .
$$


Если подняться на универсальное накрытие $D_{F}$, то легко проверить, что

$$
\widetilde{\Delta}(\alpha) K(x, y \mid \alpha)=\left(1-\alpha^{2}\right) I_{0},
$$

и поэтому

$$
\operatorname{det} K(x, y \mid \alpha)=\left(1-\alpha^{2}\right)^{r_{0}} \operatorname{det}^{-1} \widetilde{\Delta}(\alpha) .
$$

После этого утверждение теоремы 2 следует из тождества

$$
\frac{a_{+}\left(1-\alpha_{+}^{2}\right)}{a_{-}\left(1-\alpha_{-}^{2}\right)}=\frac{\alpha_{+}}{\alpha_{-}}
$$

и формулы (4.19).

Теоремы 1 и 2 устанавливают следуюшее соответствие между детерминантом матрищы рассеяния и $L$-функцией редущированного графа:

$$
\operatorname{det} S(t)=\left(\frac{\alpha_{+}}{\alpha_{-}}\right)^{r_{0}}\left(\frac{1-\alpha_{-}^{2}}{1-\alpha_{+}^{2}}\right)^{r_{0}-r_{1}} \frac{L\left(\rho, \alpha_{+}\right)}{L\left(\rho, \alpha_{-}\right)}, \quad \alpha_{+} \alpha_{-}=1 / p,
$$

где $L(\rho, \alpha)$ зависит лишш от характеров петель в редуцированном графе $F$. Соотношение (4.20) можно записать в альтернативном виде:

$$
\operatorname{det} S(t)=\left(\frac{\alpha_{+}}{\alpha_{-}}\right)^{r_{0}} \frac{\operatorname{det} \widetilde{\Delta}\left(\alpha_{-}\right)}{\operatorname{det} \widetilde{\Delta}\left(\alpha_{+}\right)} .
$$

Заметим, что зависимость от самого графа $F$, вернее, от его объема, содержится лиш в первом, достаточно тривиальном, множителе, входящем в формулы (4.20) и (4.21); все нетривиальные величины зависят только от характеристик петель графa $F$.

Поскольку $\alpha_{-} \alpha_{+}=1 / p$, то комбинация $\left(\alpha_{ \pm}\right)^{r_{0}} \operatorname{det} \widetilde{\Delta}\left(\alpha_{\mp}\right)$ имеет вид:

$$
\left(\alpha_{ \pm}\right)^{r_{0}} \operatorname{det} \widetilde{\Delta}\left(\alpha_{\mp}\right)=\operatorname{det}\left(\alpha_{ \pm} \widetilde{\Delta}\left(\alpha_{\mp}\right)\right)=\operatorname{det}\left(\alpha_{ \pm}+\frac{\alpha_{\mp}}{p} \widetilde{Q}-\frac{1}{p} \widetilde{M}_{1}\right) .
$$

ЗАмЕчАниЕ 3. Возврашаясь к условию (4.18), получим, что детерминант оператора $K\left(z, y \mid \alpha_{ \pm}\right)$невырожден (всюду кроме конечного числа точек дискретного спектра оператора $\widetilde{\Delta}(u))$. Это означает, что неоднозначность выбора функции источников $s(x)$ при этом должна быть фиксирована. Определить, каким образом происходит эта фиксация, можно, если подействовать оператором $\Delta\left(\alpha_{+}\right)$на обе части условия (4.18). В каждой внутренней вершине графа $F$ получим:

$$
\left(\alpha_{-} \Delta\left(\alpha_{+}\right) s\right)(x)=\left(\alpha_{+} \Delta\left(\alpha_{-}\right) s\right)(x)
$$

тем самым, получим, что $s(y)=c_{i} s(y)$, т.е. если только $c_{i} \neq 1$, то $s(y) \equiv 0$ во всех внутренних вершинах. Таким образом, наложение условия (4.18) позволяет полностью устранить неоднозначность выбора $s(y)$ для почти всех значений спектрального параметра $t$. (Случаи, когда такое условие невозможно наложить, соответствуют исключительньм точкам дискретного спектра, см. ниже.) 
4.2. Структура спектра. Изучим теперь спектральные свойства задачи (4.1). Эти свойства оказываются весьма близкими спектральньм свойствам задач с потенциалами шрёдингеровского типа, исследованными в работах [24].

Интерпретируем теперь задачу на собственные функции (4.1) как спектральную задачу.

ОПРЕДЕЛЕНИЕ 11. Непрерывный спектр, или спектральная зона рассеяния,это область $t^{2}<1 / p$, в которой $\alpha_{+}$и $\alpha_{-}$комплексно сопряжены друг другу, а их абсолютное значение в точности равно $1 / \sqrt{p}$; тогда получим:

$$
\lambda \in(-p-1-2 \sqrt{p},-p-1+2 \sqrt{p}) .
$$

Точки регулярного дискретного спектра - это собственные значения задачи (4.1), которые лежат вне области непрерьвного спектра (4.23) и отвечают вешественньм решениям, спадаюшим экспоненциально (как $\alpha_{+}^{n}$ ) с $\left|\alpha_{+}\right|<1 / \sqrt{p}$ и таким, что эти собственные функции не равны нулю по крайней мере на одной ветви.

Точки исключительного дискретного спектра могут возникать при любом вещественном $\lambda$. Это точки, в которых соответствующие собственные функции в точности равнь нулю вне редуцированного графа.

Первые два типа точек характерны для подавляющего большинства спектральных задач. Третий же тип специфичен именно для спектральных задач на графах, или, в более общей постановке, для дискретных пространств (его сушествование в рассматриваемой задаче было отмечено в работах [34], [39]).

Отметим, что точки исключительного дискретного спектра сушествуют далеко не для всех графов; обычно за их возникновение бывает ответственна какая-либо симметрия графа (например, симметрия $\mathbb{Z}_{2}$, см. [24]). Очевидно, что для того чтобы сушествовало такое исключительное решение, нужно, чтобы оно не только обрашалось в нуль на всех ветвях, но также занулялось во всех корневых вершинах этих ветвей, т.е. во всех внешних точках $x \in \partial F$ (и соответственно во всех внешних точках минимального редуцированного графа). Это условие не так легко формализовать; однако, в случае унитарного потенциала можно сформулировать четкий критерий существования исключительных точек дискретного спектра (см. предложение 3 ниже).

Пусть $k$ - полное число ветвей, растуших из вершин $\partial F$. Тогда для любого $\lambda$ из зоны рассеяния в случае сферических функций (т.е. функций, которые постоянны на каждом сферическом срезе каждой ветки) всегда имеется $k$-мерное подпространство собственных функций, отвечаюших данному собственному значению $\lambda$.

Зафииксировав спектральный параметр $\lambda$, введем $2 k$-мерное пространство $H^{2 k}$ асимптотических состояний - асимптотических локальных решений задачи (4.1), обладающих поведением типа сферической функции на ветвях графа $T$. На каждой ветви имеются два линейно-независимых решения, пропорциональных $\alpha_{+}^{n}$ и $\alpha_{-}^{n}$. Эти функции $\psi_{i}^{ \pm}, i=1, \ldots, k$, образуют базис в пространстве $H^{2 k}$. Тогда настоящие решения (4.14) задачи рассеяния, т.е. решения, которые могут быть продолжены на весь граф, образуют $k$-мерное подпространство $\Lambda^{k}$ пространства $H^{2 k}$.

ПроБлема 1. Можно ли развить описание в терминах лагранжевых плоскостей в случае унитарных потенциалов по аналогии со случаем потенциалов шрёдингеровского типа [24]? 
ПРЕДЛОЖЕНИЕ 2. S-матрица (4.14) унитарна в зоне рассеяния (4.23).

ДокАЗАтЕльство. Приводимое доказательство напоминает (с существенными вариациями) доказательство в [24] аналогичного утверждения для задачи рассеяния с потенциалом шрёдингеровского типа.

В качестве первого шага определим симметричную функцию $W(\varphi, \psi)$ (она будет назьваться s-вронскианом по аналогии с антисимметричнылм вронскианом работы [24]), которая отображает две функции из $C_{0}$ в функцию из $C_{1}$ по формуле:

$$
W(\varphi, \psi)=\sum_{\vec{\mu}_{x, y} \in \vec{L}}\left(\varphi^{*}(x) U_{\vec{\mu}_{x, y}} \psi(y)+\psi^{*}(x) U_{\vec{\mu}_{x, y}} \varphi(y)\right) .
$$

Если $\varphi$ и $\psi-$ две собственные функции из $\Lambda^{k}$ (по определению отвечающие одному и тому же значению спектрального параметра $\lambda$ ), то s-вронскиан есть цепь (1-цикл, возможно, с открытыми концами), т.е. для бесконечного графа получим:

$$
\partial W(\varphi, \psi)=0 .
$$

Это следует из простого вычисления:

$$
\begin{aligned}
\partial W(\varphi, \psi)(x)= & \varphi^{*}(x) \sum_{y} U_{\vec{\mu}_{x, y}} \psi(y)+\psi^{*}(x) \sum_{y} U_{\vec{\mu}_{x, y}} \varphi(y) \\
& -\varphi^{*}(y) \sum_{y} U_{\vec{\mu}_{y, x}} \psi(x)-\psi^{*}(x) \sum_{y} U_{\vec{\mu}_{y, x}} \varphi(x) \\
= & \varphi^{*}(x)\left(M_{1} \psi\right)(x)-\left(\varphi^{*}(x)\left(M_{1} \psi\right)(x)\right)^{*} \\
& +\psi^{*}(x)\left(M_{1} \varphi\right)(x)-\left(\psi^{*}(x)\left(M_{1} \varphi\right)(x)\right)^{*}
\end{aligned}
$$

Используя затем соотношение $M_{1} \varphi=(Q+1-\lambda) \varphi$ и учитьвая, что $Q$ и $\lambda$ - эрмитовы операторы, получим, что правая часть (4.25) обращается в нуль.

Таким образом, s-вронскиан двух решений должен быть цепью. Обрежем теперь ветви полного (бесконечного) графа на некотором расстоянии $n$ от редуцированного граффа $F$ (это расстояние не обязано совпадать для разных ветвей; технически удобно только полагать это расстояние одинаковым для всех точек обрезания на одной ветке), тем самым получив некоторый конечный граф $T_{\text {cut }}$. При этом следующая полная сумма исчезает в силу условия 1-цикла:

$$
\sum_{x \in \partial T_{\text {cut }}} \partial W=0
$$

Здесь сумма берется по всем граничным точкам $x \in \partial T_{\text {cut }}$, а функция $\partial W$ ограничена на граф $T_{\text {cut }}$.

На $H^{2 k}$ удобно ввести билинейное спаривание:

$$
\langle\langle\psi, \varphi\rangle\rangle \equiv \sum_{x \in \partial T_{\mathrm{cut}}} \partial W(\psi, \varphi)
$$

на границе $T_{\text {cut }}$ для двух произвольных асимптотических векторов из $H^{2 k}$. 
Рассуждения в начале раздела 4 показывают, как правильным образом устранить зависимость от калибровочных потенциалов на всех внешних ветвях. Тогда функции $\psi_{i}^{ \pm}-$это просто степени $\alpha_{ \pm}: \psi_{i}^{ \pm}=\alpha_{ \pm}^{n}$ на $i$-й ветви и равны нулю вне ее. Теперь, используя тождества $\alpha_{+} \alpha_{-}=1 / p$ и $\alpha_{-}=\alpha_{+}^{*}$, вьполненные в зоне рассеяния, легко найти, что

$$
\left\langle\left\langle\psi_{i}^{ \pm}, \psi_{j}^{ \pm}\right\rangle\right\rangle= \pm \delta_{i, j} \frac{\alpha_{-}-\alpha_{+}}{2 p} \equiv \pm \delta_{i, j}\left\langle\left\langle\psi^{+}, \psi^{+}\right\rangle\right\rangle
$$

$$
\left\langle\left\langle\psi_{i}^{ \pm}, \psi_{j}^{\mp}\right\rangle\right\rangle \equiv 0 .
$$

Заметим снова, что для любых двух решений $\psi$ и $\varphi$ спектральной задачи (4.1), заданных на полном графе, выражение для $\partial W(\psi, \varphi)$ в точности совпадает с $\langle\langle\psi, \varphi\rangle\rangle$, где надо учитывать только асимптотические состояния. Рассмотрим теперь собственные функции $\Psi_{i} \equiv \psi_{i}^{+}-\sum_{j} s_{i, j} \psi_{j}^{-}$(ср. с формулой (4.14)). ${ }^{3}$ Тогда для любых двух таких функций получим:

$$
0=\left\langle\left\langle\Psi_{i}, \Psi_{j}\right\rangle\right\rangle \equiv\left\langle\left\langle\psi^{+}, \psi^{+}\right\rangle\right\rangle\left(\delta_{i, j}-\sum_{k} s_{i, k}^{*} s_{j, k}\right),
$$

и поскольку $\left\langle\left\langle\psi^{+}, \psi^{+}\right\rangle\right\rangle \neq 0$, то $S$-матрища унитарна. Предложение доказано. ${ }^{4}$

Интересно, что можно выяснить вопрос о существовании исключительного дискретного спектра, исследуя саму $L$-функцию.

ПРЕДЛОЖЕНИЕ 3. Исключительный дискретный спектр возникает тогда и только тогда, когда $\exists \alpha_{+}, \alpha_{-}: \alpha_{+} \alpha_{-}=1 / p$ и L-функиия имеет полюсы как $в$ точке $\alpha_{+}$, так и в точке $\alpha_{-}$.

НАБРОСОК ДОКАЗАТЕЛЬСТВА. Ищется экспоненциальное решение спектральной задачи (4.1) такое, что одна из волн - запаздьвающая или отраженная - в точности равна нулю во всех вершинах графа $T$ (в том числе и во внутренних точках $F$ ). Отсюда сразу следует, что существует такой вектор (функция источника) $f_{0} \in \widetilde{C}_{0}$, что

$$
\Delta\left(\alpha_{+}\right) f_{0}=0 .
$$

В этой точке нелокальньй оператор $\widehat{K}$,

$$
\widehat{K} f(x)=\sum_{y \in F} K(x, y \mid \alpha) f(y), \quad f \in \widetilde{C}_{0},
$$

который обратен $\widetilde{\Delta}(\alpha)$ при общих значениях $\alpha$, становится плохо определенньм.

Если $f_{0}-$ нулевой вектор $\widetilde{\Delta}(\alpha)$, то характеристическое уравнение на $\alpha$ имеет вид:

$$
\operatorname{det} \widetilde{\Delta}\left(\alpha_{+}\right)=0,
$$

\footnotetext{
${ }^{3}$ Используются сокращенные мультииндексные обозначения.

${ }^{4}$ Возврашаясь к формуле (4.20), получим, что детерминант $S$-матрицы унитарен, если только $\alpha_{ \pm}$попадают в зону рассеяния; это разумеется находится в согласии с предложением 2.
} 
и если одновременно $\widetilde{\Delta}\left(\alpha_{-}\right) \neq 0$, то ввиду теоремы 2 , соответствующая $S$-матрища имеет сингулярность (нуль или полюс), что показывает, что при таком значении $\lambda$ существует такое решение спектральной задачи (4.1), что в нем одна (и только одна) из падающей и отраженной волн тождественно зануляется (ср. [34], [39]).

Таким образом, если не происходит сокрашений между числителем и знаменателем в формуле (4.20), т.е. условие предложения не выполнено, то дискретньй спектр исчерпьвается регулярньми собственными функциями и исключительный спектр пуст.

Напротив, если такие полюсы $L$-функции имеют место, т.е. условие предложения вьполнено, то регулярные точки дискретного спектра не задают всех возможных решений характеристического уравнения; при этом решения, которые зануляются как в числителе, так и в знаменателе в формуле (4.20), должны отвечать исключительньм дискретным собственным значениям.

ПроБлемА 2. Превратить этот набросок доказательства в формальное доказательство.

\section{5. Примеры. Алгоритм для вычисления $L(1, u)$}

Для простоты в дальнейшем предполагается, что $F=D(\Gamma) / \Gamma$. Большинство доказательств в этом и следуюшем разделах представляют собой простые упражнения в линейной алгебре и будут опушены.

ПримеР 1. Граф Т без внешних ветвей. Рассмотрим предельньй случай рассматриваемой конструкции, когда граф $T$ в точности совпадает с $F$. В этом случае рассеивание отсутствует, $q(z) \equiv p$ и можно получить соотношения обратимости для $\Delta(u)$. Если $\alpha_{-} \alpha_{+}=1 / p$, то получим:

$$
\begin{aligned}
\operatorname{det}^{-1}\left[\left(1+p \alpha_{+}^{2}\right) I-\alpha_{+} M_{1}\right] & =\operatorname{det}^{-1}\left[1+\frac{1}{p \alpha_{-}^{2}}-\frac{1}{p \alpha_{-}} M_{1}\right] \\
& =\left(\alpha_{-}^{2} p\right)^{r_{0}} \operatorname{det}^{-1}\left[\left(1+p \alpha_{-}^{2}\right) I-\alpha_{-} M_{1}\right] \\
& =\frac{\alpha_{-}^{r_{0}}}{\alpha_{+}^{r_{0}}} \operatorname{det}^{-1}\left[\left(1+p \alpha_{-}^{2}\right) I-\alpha_{-} M_{1}\right]
\end{aligned}
$$

и из соотношений (4.21) и (5.1) легко получить, что полная матрища рассеяния $C \equiv 1$. Таким образом, матрица $C$ тривиальна и не зависит вообще от формы редуцированного или в данном случае полного графа $T=F$. Это находится в согласии с тем фактом, что в этом случае все точки спектра суть точки исключительного дискретного спектpa.

Пример 2. Случай одной петли $(g=1)$. В случае, когдаграф $T$ содержит только одну петлю, а характеры групшы Г тривиальны, $\chi(u)=1, L$-функция имеет вид:

$$
L(1, u)_{g=1}=\frac{1}{\left(1-u^{n}\right)^{2}},
$$

поскольку группа $\Gamma_{1}$ в этом случае содержит лишь два примитивных элемента $\gamma$ и $\gamma^{-1}$ с одной и той же длиной $l(\gamma)=l\left(\gamma^{-1}\right)=n$. 
5.1. Две петли. Общая структура $L(1, u)$. Опишем алгоритм для вычисления $L(u) \equiv L(1, u)$ на произвольном графе. Обозначим через $l_{i}$ длины ребер некоторого графа $F$ и рассмотрим величины $a_{i} \equiv u^{l_{i}}$. Тогда из теоремы 1 получим, что $L^{-1}(u)=\operatorname{det}\left(1-u T_{1}\right)=P_{2 r_{1}}$ представляется в виде многочлена степени $2 \sum l_{i}$ по переменной $u$. Этот многочлен, очевидно, представим как многочлен от нескольких переменных $a_{i}$ (поскольку все замкнутые геодезические строятся из отрезков, соединяюших трех- и более валентные вершины $F$ ), и величины $a_{i}$ могут интерпретироваться как формальные переменные. Предполагая, что одно из чисел $l_{i}$ превосходит сумму всех остальных, получим с необходимостью, что максимальная степень многочлена $P_{2 r_{1}}(u)$ по каждой из переменных $a_{i}$ меньше или равна двум. Поэтому, $L^{-1}(u)=P_{2}\left(\left\{a_{i}\right\}\right)$. Коэффициенты этого многочлена - целые числа, и из соотношения

$$
L^{-1}(u)=\operatorname{det}\left(1-u T_{1}\right)=\left(1-u^{2}\right)^{g-1} \operatorname{det} \Delta(u)
$$

следует, что $L^{-1}(u)$ имеет нуль порядка как минимум $g-1$ в точке $u=-1$ и нуль в точности порядка $g$ при $u=1$. Последнее следует из соотношения $\left.\operatorname{det} \Delta(u)\right|_{u=1}=0$, вьполненного для произвольного графа, и из соотношения

$$
\left.\frac{\partial}{\partial u} \operatorname{det} \Delta(u)\right|_{u=1}=\# \text { максимальных деревьев }
$$

(см. [40], где было отмечено, что производная $\Delta(u)$ в точке $u=1$ может быть выражена по формуле Кирхгофа через полное число максимальных связных подграфов типа дерева, т.е. подграфов без циклов, содержашихся в графе $F$, и тем самым это число отлично от нуля).

Рассмотрим, например, случай графа с двумя петлями. Нарисовать такой связный граф̆ можно тремя способами (см. рис. 4, а), б) и в)).

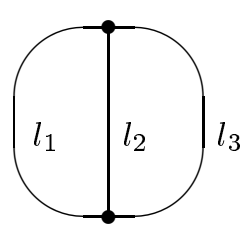

a)

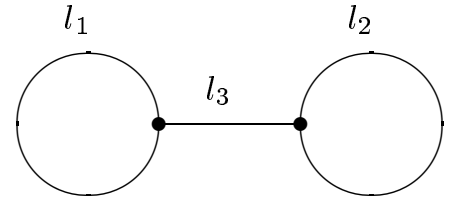

б)

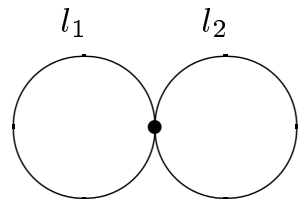

в)

Рис. 4. Три возможных графа для $g=2$

В работе [14] показано, как можно сравнительно легко вычислить соответствуюшие $L$-функции, используя вышеприведенные соображения. Ответ имеет вид:

$$
\begin{aligned}
& \text { случай а) } L_{(a)}^{-1}=\left(1-\sum_{i<j} a_{i} a_{j}-2 a_{1} a_{2} a_{3}\right)\left(1-\sum_{i<j} a_{i} a_{j}+2 a_{1} a_{2} a_{3}\right), \\
& \text { случай б) } L_{(b)}^{-1}=\left(1-a_{1}\right)\left(1-a_{2}\right)\left[\left(1-a_{1}\right)\left(1-a_{2}\right)-4 a_{3}^{2} a_{1} a_{2}\right], \\
& \text { случай в) } L_{(c)}^{-1}=\left(1-a_{1}\right)\left(1-a_{2}\right)\left[\left(1-a_{1}\right)\left(1-a_{2}\right)-4 a_{1} a_{2}\right] .
\end{aligned}
$$

(Заметим, что случай в) получается из а) или б), если положить $a_{3}=1$.) 


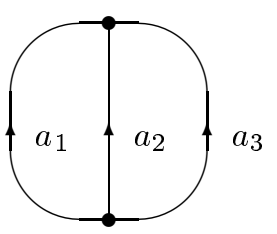

a)

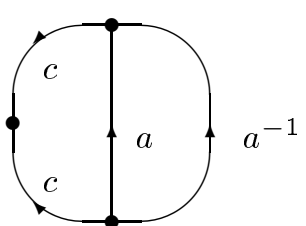

б)

Рис. 5. Примеры граффов с эрмитовым и унитарным потенщиалами

ПРИмеР 3. Сравнение әрмитовых и унитарньх потенииалов. Рассмотрим $L$-функции и соответствуюшие детерминанты для двух графов, изображенных на рис. 5, где потенциалы предполагаются абелевыми.

Выпишем ответы для эрмитова и унитарного потенциалов (в последнем случае $a_{i} \rightarrow a_{i}^{-1}$ при обращении ориентации ребра) в случае а). Для эрмитова потенциала получим:

$$
\begin{gathered}
\operatorname{det} \Delta(u)=\left(1+2 u^{2}\right)^{2}-s^{2} u^{2}, \quad s \equiv a+b+c, \\
\operatorname{det}(1-T(u))=1-2 u^{2}(a b+b c+c a)+u^{4}(a b b+b c+c a)^{2}-4 u^{6} a^{2} b^{2} c^{2},
\end{gathered}
$$

в то время как для унитарного потенциала эти две величины, разумеется, связаны:

$$
\begin{gathered}
\operatorname{det} \Delta(u)=\left(1+2 u^{2}\right)^{2}-s \tilde{s} u^{2}, \quad s \equiv a+b+c, \quad \tilde{s} \equiv a^{-1}+b^{-1}+c^{-1}, \\
\operatorname{det}(1-T(u))=\left(1-u^{2}\right) \operatorname{det} \Delta(u) .
\end{gathered}
$$

Случай б) на рис. 5 интересен потому, что это как раз тот случай, когда исключительньй дискретньй спектр при $p=2$ непуст при любом $a$ (достаточно поместить равные по абсолютной величине положительные и отрицательные источники в соответствующих верхней и нижней вершинах на рис. 5 б); тогда собственная функция тождественно обрашается в нуль в средней точке). Для $L$-функции получим:

$$
L(u)=\left(1+u x+2 u^{2}\right)\left(1-u x+u^{2}-u^{3} x+2 u^{4}\right), \quad x \equiv a+a^{-1}, \quad p=2,
$$

т.е. условие предложения 3 вьполнено при любом $a$, поскольку произведение двух корней квадратного уравнения в этом случае всегда равно $1 / 2 \equiv 1 / p$.

\section{6. Пространства Тейхмюллера и графы}

В этом разделе исследуются пространства Тейхмюллера $\mathscr{T}^{h}$ - пространства комплексных структур на (возможно открытых) римановых поверхностях $S$ с дырками (выколотыми точками) по модулю диффеоморфизмов, гомотопически эквивалентных тождественному.

В окрестности граничной компоненты комплексная структура может быть изоморфна как комплексное многообразие либо кольцу (в случае дырки), либо проколотому диску (для выколотой точки). 
Описание в терминах графов для таких поверхностей было предложено Пеннером [19] и развито Фоком [20] и Кашаевым [41]. Вместо самого пространства Тейхмюллера $\mathscr{T}^{h}(S)$ графы описывают его конечное накрытие $\mathscr{T}^{H}(S)$. Точка пространства $\mathscr{T}^{H}(S)$ определяется точкой из $\mathscr{T}^{h}(S)$ и ориентациями всех тех дырок поверхности $S$, которые не являются выколотыми точками. (Это накрытие разветвляется надподпространством поверхностей с выколотыми точками.)

Хорошо известно, что ориентированная двумерная поверхность с отрицательной эйлеровой характеристикой может быть непрерывно конформно преобразована к поверхности с постоянной отрицательной кривизной. Теорема униформизации Пуанкаре утверждает, что всякая комплексная поверхность $S$ постоянной отрицательной кривизны (в дальнейшем полагаемой равной -1 ) представляется как фактор верхней полуплоскости $\mathbb{H}_{+}$с гиперболической метрикой $d s^{2}=d z d \bar{z} /(\operatorname{Im} z)^{2}$ относительно действия дискретной фуксовой подгруппы $\Delta(S)$ группы автоморфизмов $P S L(2, \mathbb{R})$ :

$$
S=\mathbb{H}_{+} / \Delta(S)
$$

Геодезические в гиперболической метрике представляют собой или полуокружности с концами на вешественной прямой $\mathbb{R}$, или вертикальные полупрямые; все точки границы $\mathbb{R}$ лежат на бесконечном расстоянии друг от друга и от любой внутренней точки.

Каждый гиперболический гомотопический класс замкнутых кривых $\gamma$ содержит единственную замкнутую геодезическую длины $l(\gamma)=\left|\log \lambda_{1} / \lambda_{2}\right|$, где $\lambda_{1}$ и $\lambda_{2}-$ (различные) собственные значения элемента из $P S L(2, \mathbb{R})$, который отвечает классу $\gamma$.

Начиная с работы Штребеля [42], жирные, или ленточные, графы используются для координатизации пространств модулей и пространств Тейхмюллера. Простое и явное описание, используемое в данной работе, восходит к работе [20].

УТВЕРЖДЕНИЕ 1. Для данного конечного ленточного графа $T$ с трехвалентными вершинами, имеющего род (число ручек) $g$ и число выколотых точек (компонент связности) $n$, существует взаимно однозначное соответствие

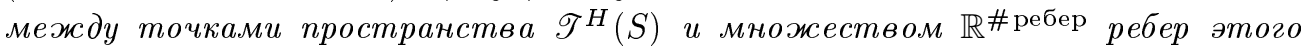
графа с заданными на этих ребрах вещественныци числами (длинами).

Сушествует явная конструкция, позволяюшая построить фуксову группу $\Delta(S) \subset$ $P S L(2, \mathbb{R})$, которая отвечает данному набору чисел на ребрах графа $T \in T(S)$, такую, что $S=\mathbb{H}_{+} / \Delta(S) .^{5}$ Для этого необходимо задать правило, по которому каждому элементу из группы $P_{\gamma} \in P S L(2, \mathbb{R})$ ставился бы в соответствие элемент фундаментальной групшы $\gamma \in \pi_{1}(S)$.

Во-первых, поставим в соответствие каждому ребру $\alpha$ матрицу $X_{Z_{\alpha}} \in P S L(2, \mathbb{R})$ преобразования Мёбиуса:

$$
X_{Z_{\alpha}}=\left(\begin{array}{cc}
0 & -\mathrm{e}^{Z_{\alpha} / 2} \\
\mathrm{e}^{-Z_{\alpha} / 2} & 0
\end{array}\right)
$$

\footnotetext{
${ }^{5}$ Заметим, что $\pi_{1}(S)$ изоморфна $\pi_{1}(\Gamma)$.
} 
Далее, для того чтобы параметризовать путь по ребрам графа, вводятся матришы “правых" и “левых" поворотов:

$$
R=\left(\begin{array}{cc}
1 & 1 \\
-1 & 0
\end{array}\right), \quad L \equiv R^{2}=\left(\begin{array}{cc}
0 & 1 \\
-1 & -1
\end{array}\right)
$$

Пространства $C_{0}$ и $C_{1}$ - это пространства функций, принимающих значения в фундаментальном (размерности два) представлении групшы $P S L(2, \mathbb{R})$.

“Операторы правых и левых сдвигов", $R_{Z}$ и $L_{Z}$, имеют вид:

$$
\begin{aligned}
R_{Z} & \equiv R X_{Z}=\left(\begin{array}{cc}
\mathrm{e}^{-Z / 2} & -\mathrm{e}^{Z / 2} \\
0 & \mathrm{e}^{Z / 2}
\end{array}\right), \\
L_{Z} & \equiv L X_{Z}=\left(\begin{array}{cc}
\mathrm{e}^{-Z / 2} & 0 \\
-\mathrm{e}^{-Z / 2} & \mathrm{e}^{Z / 2}
\end{array}\right) .
\end{aligned}
$$

Оператор $T(u)$ действует на пространстве $C_{1}$ следующим образом. Обозначим через $\vec{e}_{R}$ (соответственно $\vec{e}_{L}$ ) ориентированное ребро, в которое ориентированное ребро $\vec{e}_{Z}$ отображается естественным образом при правом (соответственно левом) повороте при движении вдоль последовательных ребер некоторого пути. Для $u \in \mathbb{C}$ и вектора $\vec{e} \in \vec{L}$ получим:

$$
T(u) v_{\vec{e}} \cdot \vec{e}_{Z}=v_{\vec{e}_{R}} \cdot \vec{e}_{R}+v_{\vec{e}_{L}} \cdot \vec{e}_{L}
$$

где

$$
v_{\vec{e}_{R}}=u R_{z} v_{\vec{e}} \text { и } v_{\vec{e}_{L}}=u L_{z} v_{\vec{e}}
$$

Геодезическая -это замкнутый примитивный путь в графе $T$. Каждому такому пути ставится в соответствие произведение матрищ

$$
P_{Z_{1} \cdots Z_{n}}=L_{Z_{n}} L_{Z_{n-1}} R_{Z_{n-2}} \cdots R_{Z_{2}} L_{Z_{1}}
$$

где матрищы $L_{Z_{i}}$ или $R_{Z_{i}}$ вставляются в зависимости от того, в какую сторону - правую или левую - поворачивает путь на графе на соответствуюшем шаге.

Матрицы $L$ и $R$ - это потенциалы кручения из определения 5. Однако, можно модифицировать исходньй граф̆ $T$, рассоединяя в каждой вершине ребра, входящие в нее, и соединяя снова указанные ребра тремя новыми “короткими" ребрами, образующими треугольник, и сразу же стирая одно (все равно какое) из этих новых ребер; при этом число петель в графе остается постоянным (см. рис. 6).
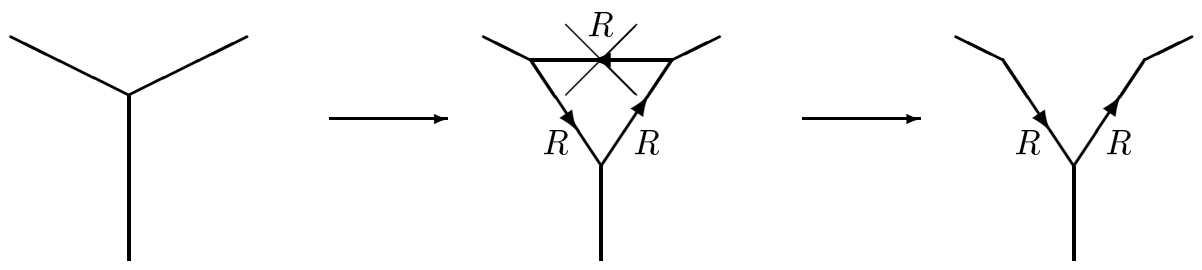

Рис. 6. "Раздувание" вершин исходного графа 
После такого раздутия вершин матрицы $L$ и $R$ становятся матрицами потеншиалов, сосредоточенных на "коротких" ориентированных ребрах получаюшегося граффа $\widetilde{T}$.

ПрЕДЛОЖЕНИЕ 4 [20]. Существует взаимно однозначное соответствие между әлементами множества всех примитивных замкнутых путей $\left\{P_{Z_{1} \cdots Z_{n}}\right\}$ графа $T$ и замкнутыми геодезическими $\{\gamma\}$ на соответствующей римановой поверхности. Более того, длина $l(\gamma)$ геодезической в метрике Пуанкаре задается соотношением:

$$
G(\gamma) \equiv 2 \operatorname{ch}(l(\gamma) / 2)=\operatorname{tr} P_{Z_{1} \cdots Z_{n}}
$$

ЗАмЕчАниЕ 4. Поскольку обе матрицы (6.3) и (6.4) имеют вид $\left\{\begin{array}{ll}(+) & (-) \\ (-) & (+)\end{array}\right\}$, то и любое произведение этих матриц имеет тот же вид; более того, в диагональных членах таких произведений все слагаемые входят с положительным, а в антидиагональных - с отрицательным знаком. Тогда для замкнутой геодезической, гомотопной дыгрке (геодезической, идушей по грани), получим: $l(\gamma)=\left|\sum_{i \in I} Z_{i}\right|$, где суммирование ведется по всем граничньм ребрам данной грани (взятым с нужными кратностями).

ОПРЕДЕЛЕнИЕ 12. $L$-функция Ихары-Сельберга $L(u)$ ленточного графа $T$ имеет вид:

$$
L(u)=\prod_{\{\varpi\}} \operatorname{det}^{-1}\left(I-u^{n} P_{z_{1} \cdots z_{n}}\right)
$$

(ср. (3.1)), где произведение берется по всем простым замкнутым путям и $n$ - длина пути, измеряемая в единицах расстояния на универсальном накрьваюшем дереве.

Теперь из теоремы 1 следует, что $L$-функция Ихары-Сельберга (6.6) - это рациональная функция относительно переменных $\mathrm{e}^{Z_{i} / 2}$ и $u$ и

$$
L(u)=\operatorname{det}^{-1}(I-T(u))=u^{r_{0}} \operatorname{det}^{-1} \Delta(u) .
$$

Произведение в формуле (6.6) сходится абсолютно в круге $|u|<\frac{1}{2} \min \left\{\left|\mathrm{e}^{-Z_{i}}\right|\right\}$. Более того, из теоремы 1 следует, что оно допускает однозначное аналитическое продолжение на всю комплексную плоскость $\mathbb{C}$ кроме конечного числа сингулярных точек.

6.1. Формула следа Сельберга и распределение геодезических. В случае замкнутой римановой поверхности постоянной отрищательной кривизны были получены сильные результаты относительно распределения замкнутых геодезических по длинам (см. [43], [44]).

ОпРЕДЕЛЕнИЕ 13. Дзета-функиия для замкнутых геодезических на (проколотой) римановой поверхности $S$ имеет вид:

$$
\zeta(s)=\prod_{n}\left(1-\mathrm{e}^{-\operatorname{sh} l_{n}}\right)^{-1},
$$

где $h$ - константа, задаваемая асимптотическим распределением длин геодезических.

Если $\pi(T)$ - это число замкнутых (примитивных) геодезических, длины которых не превышают $T$, то можно доказать справедливость следуюшего утверждения (см., например, [43], [44]). 
УТВЕРЖДЕНИЕ 2. Существует такая константа $h$, что

$$
\lim _{T \rightarrow \infty} \frac{\pi(T)}{\mathrm{e}^{h T} / h T}=1
$$

Доказательство утверждения 2 основано на аналитических свойствах $\zeta$-функции.

ПРЕДЛОЖЕНИЕ 5 [43]. Функиия $\zeta(s)$ может бъть продолжена на $\mathbb{C}$ как мероморфная функиия такая, что:

а) $\zeta(s)$ не имеет ни нулей, ни полюсов в области $\operatorname{Re} s \geqslant 1, s \neq 1$;

б) $\zeta(s)$ имеет простой полюс в точке $s=1$.

Существуют два альтернативных доказательства предложения 5: одно из них основано на знаменитой формуле следа Сельберга, которая в простейшей формулировке имеет следуюший вид.

ПРЕДЛОЖЕНИЕ 6 (доказательство см. [5], [45]). Для заданного оператора Бельтрами-Лапласа $\Delta$, действующего на гиперболическом пространстве с метрикой постоянной отрицательной кривизны и представляющего собой линейный (неограниченныц ) оператор второго порядка в частных производных с дискретным спектром $0=\lambda_{0}<\lambda_{1} \leqslant \lambda_{2} \leqslant \cdots$, и полного набора замкнутых геодезических $c$ длинами $\left\{l_{n}\right\}$ на римановой поверхности, формула следа Сельберга связывает эти две последовательности вещественных чисел $\left\{\lambda_{n}\right\}$ u $\left\{l_{n}\right\}$ :

$$
\sum_{n} \hat{f}\left(\sqrt{\lambda_{n}-1 / 4}\right)+\int f d t=\sum_{n} c_{n}\left(f\left(l_{n}\right)\right)+2 \frac{\operatorname{Area}(V)}{4 \pi} \int_{-\infty}^{\infty} r \operatorname{th}(r) \hat{f}(r) d r
$$

где $f: \mathbb{R} \rightarrow \mathbb{R}$ есть $C^{\infty}$-функиия с компактным носителем, $\hat{f}$-ее преобразование Фурье $u$

$$
c_{n}\left(f\left(l_{n}\right)\right)=\sum_{k=1}^{\infty} \frac{l_{n} f\left(k l_{n}\right)}{\operatorname{sh}\left(k l_{n} / 2\right)}
$$

Для компактной поверхности Area $(V) / 2 \pi=2(g-1)$.

Альтернативное доказательство в [43] использует методы символьной динамики и весьма близко по духу к описанию в терминах комбинаторных графов. В заключение этого раздела отметим две проблемы.

Проьлема 3. Связать описание в терминах графов дзета- (или $L$-) функции (3.1) на пространстве Тейхмюллера со стандартной дзета-функцией (6.8).

ПроБлЕмА 4. Известно несколько явных формул, используюших геодезические на открытых римановых поверхностях. Отметим работу [46], в которой для проколотого тора и проколотых штанов (a pair of pants) были получены формулы, в которых участвуют не все, а только простые (т.е. несамопересекающиеся) геодезические. Сушествуют ли обобшения этих формул на случай обших поверхностей с дырками? 
6.2. Классические проективные (модулярные) преобразования. В работе [20] были построены проективные преобразования графа, соответствующие преобразованиям из класса отображений (модулярным преобразованиям). Они соответствуют естественным операциям над множеством графов, называемым флипами или движениями Уайтхеда, которые задают элементарные переходы между графами (более точно, между соседними клетками симплициального комплекса, клетки высшей размерности которого находятся во взаимно однозначном соответствии с комбинаторными типами трехвалентных ленточных графов данного (риманова) рода $g$ и $n$ ). Соответствуюшее преобразование переменных $Z_{\alpha}$ нелинейно (явные выражения см. в [20]-[22]), однако длины геодезических при этом оказываются модулярно-инвариантными функциями.

Лемма 3. В точке $u=1$ функция $L(u)$ модулярно инвариантна, т.е. не зависит от частного выбора параметризующего графа.

Заметим, однако, что длина геодезической $n$, измеряемая в терминах граф̆а (число ребер), изменяется при модулярных преобразованиях, и пока что не удалось определить полньй аналог дзета-функции (6.8) непосредственно в терминах графа. Отметим, что конструкции типа $L$-функции (6.6) тем не менее встречаются в символьной динамике, где часто вводятся дополнительные модулярно-неинвариантные разбиения римановых поверхностей, которые соответствуют разбиению поверхностей с помошью графов (см. гл. 5 и 6 сборника [44]).

ЗАмЕЧАниЕ 5 . Что касается функции $L^{-1}(u)$, заметим, что детерминант в формуле (6.6) - многочлен Лорана не выше второго порядка по $\mathrm{e}^{ \pm z_{i} / 2}$ для каждого $z_{i}$. Более того, из леммы 2 следует, что модулярно-инвариантное выражение для $L(1)$ может содержать зависимость только от модулярных инвариантов - "периметров" дырок. Это накладывает жесткие ограничения на возможный вид $L^{-1}(1)$. Например, для модулярных пространств $M_{g, 1}$ римановых поверхностей с одним проколом (дыркой) имеется лиш один такой параметр $p_{1}$. Учитывая глобальную симметрию $x_{i} \rightarrow-x_{i}$, получим, что

$$
L_{g, 1}^{-1}(1)=a \operatorname{ch}\left(p_{1}\right)+b \operatorname{ch}\left(p_{1} / 2\right)+c, \quad a+b+c=0,
$$

где только коэффициенты $a, b$ и $c$ зависят от рода поверхности.

ПримеР 4. 1. В пространстве модулей $M_{1,1}$ (тор с одним проколом) имеются три переменные $x, y$ и $z$ и один модулярный инвариант $p_{1}=x+y+z$,

$$
L_{1,1}^{-1}(1)=-8(\operatorname{ch}(x+y+z)-1) .
$$

2. В пространстве модулей $M_{0,3}$ (сфера с тремя проколами) имеются опять-таки три переменные $x, y$ и $z$, но и три модулярно-инвариантные комбинации $x+y=p_{3}$, $x+z=p_{2}, y+z=p_{1}$. Тогда

$$
L_{0,3}^{-1}(1)=-2^{5}\left(\operatorname{ch}\left(\frac{x+y}{2}\right)-1\right)\left(\operatorname{ch}\left(\frac{x+z}{2}\right)-1\right)\left(\operatorname{ch}\left(\frac{y+z}{2}\right)-1\right) .
$$




\section{7. Заключение}

Основной результат данной статьи - это формула (4.20), устанавливаюшая связь между $L$-функциями и данными задачи рассеяния. Эта формула аналогична формуле следа Сельберга в случае дискретных некомпактных однородных пространств (графов).

Интересно продолжить исследование спектральных свойств $S$-матрицы, изучаемых в разделе 4 , особенно в случае нетривиальных унитарных потенциалов и потенциалов шрёдингеровского типа. В этой связи встает задача разработки аналогичных методов для симплициальных комплексов старших размерностей в духе работы [47].

Можно попытаться применить методы, развиваемые в данной статье, к случаю непрерывных открытых поверхностей в надежде получить аналогичные формулы для процессов непрерьвного рассеяния.

Наконец, представляется вероятным, что в подобных процессах рассеяния скрыта некоторая $r$-матричная структура. Встает проблема дать аналогичное описание (нелинейных) дискретных интегрируемых моделей.

После окончания данной работы вьшла статья [48], в которой в формализме функционального интеграла исследовались магнитные потоки на похожих бесконечных графаx.

Я благодарен С. П. Новикову и участникам его семинара в Математическом институте им. В. А. Стеклова РАН за полезные обсуждения и предложение написать данную работу.

\section{СПИСОК ЛИТЕРАТУРЫ}

[1] Selberg A. Harmonic analysis and discontinuous groups in weakly symmetric Riemannian spaces, with applications to Dirichlet series // J. Indian Math. Soc. 1956. V. 20. P. 47-87.

[2] Colin De Verdière Y. Spectre du laplacien et longueur des géodésiques fermées II // Compositio Math. 1973. V. 27. P. 159-184.

[3] Hejhal D. A. The Selberg trace formula and the Riemann zeta function // Duke Math. J. 1976. V. 43. P. 441-482.

[4] Cartier P., Voros A. Une nouvelle interprétation de la formule des traces de Selberg // C. R. Acad. Sci. Paris Sér. I Math. 1988. V. 307. P. 143-148.

[5] Лакс П.Д., Филлипс Р.С. Теория рассеяния для автоморфных функций. М.: Мир, 1979.

[6] Павлов Б. С., Фаддеев Л. Д. Теория рассеяния и автоморфные функции // Зап. научн. семин. ЛОМИ. 1972. Т. 27. С. 161-193.

[7] Венков А.Б. Спектральная теория автоморфных функций, дзета-функция Сельберга и некоторые проблемы аналитической теории чисел и математической физики // УМН. 1979. T. 34. № 3. C. $69-135$.

[8] Ihara Y. On discrete subgroup of the two by two projective linear group over $p$-adic field // J. Math. Soc. Japan. 1966. V. 18. № 3. P. 219-235.

[9] Hashimoto K.I. Zeta functions of finite graphs and representations of $p$-adic groups // Adv. Stud. Pure Math. 1989. V. 15. P. 211-280.

[10] Hashimoto K. I. On zeta and $L$-functions of finite graphs // Internat. J. Math. 1990. V. 1. № 4. P. 381-396.

[11] Bass H. The Ihara-Selberg zeta functions of a tree lattice // Internat. J. Math. 1992. V. 3. № 6. P. 717-797.

[12] Ихара Я. Дискретные подгруппы $P L\left(2, k_{p}\right) / /$ Математика: сб. перев. 1968. Т. 12. № 5 . C. $131-138$. 
[13] Chekhov L. L-functions in scattering on $p$-adic multiloop surfaces // J. Math. Phys. 1995. V. 36. № 1. P. 414-425.

[14] Chekhov L. O. Scattering on univalent graphs from $L$-function viewpoint // TM 1995. T. 103. C. $489-506$.

[15] Манин Ю.И. р-адические автоморфные функции // Совр. пробл. матем. Т. 3. М.: ВИНИТИ, 1974. С. 5-92.

[16] Serre J. P. Trees. Berlin: Springer-Verlag, 1980.

[17] Gerritzen L., van der Put M. Schottky Groups and Mumford Curves. Berlin: Springer-Verlag, 1980. (Lecture Notes in Math. V. 817.)

[18] Chekhov L.O., Mironov A.D., Zabrodin A.V. Multiloop calculations in $p$-adic string theory and Bruhat-Tits trees // Comm. Math. Phys. 1989. V. 125. P. 675-711.

[19] Penner R. C. The decorated Teichmüller space of Riemann surfaces // Comm. Math. Phys. 1988. V. 113. P. 299-339.

[20] Fock V.V. Combinatorial description of the moduli space of projective structures // Preprint hep-th/9312193.

[21] Чехов Л.О., Фок В. В.Квантовые модулярные группы, соотношение пятиугольника и геодезические // Труды МИАН. 1999. Т. 226. С. 164-179.

[22] Фок В. В., Чехов Л. О. Квантовые пространства Тейхмюллера // ТМФ. 1999. Т. 120. C. $511-528$.

[23] Новиков С. П. Оператор Шрёдингера на графах и топология // УМН. 1997. Т. 52. №6. C. $177-178$.

[24] Novikov S. P. Schrödinger operators on graphs and symplectic geometry // Special Issue in honour of V. I. Arnold, on the occasion of his 60th birthday. Toronto: Fields Institute, 1999.

[25] Долбилин Н. П., Зиновьев Ю. М., Штанько М. А., Штогрин М. И. Комбинаторный метод Каца-Уорда // УМН. 1998. Т. 53. № 6. С. 247-248.

[26] Долбилин Н. П., Зиновьев Ю. М., Мищенко А. С., Штанько М. А., Штогрин М. И. Детерминант Каца-Уорда // Труды МИАН. 1999. Т. 225. С. 177-194.

[27] Венков А. Б., Никитин А. М. Формула следа Сельберга, графы Рамануджана и некоторые проблемы математической физики // Алгебра Анал. 1993. Т. 5. № 3. С. 1-76.

[28] Vaksman L. L., Korogodskii L. I. Harmonic analysis on quantum hyperboloids // Препринт 90-27. Киев: Ин-т теор. физ., 1990.

[29] Freund P. G.O. Scattering on $p$-adic and on adelic symmetric spaces // Phys. Lett. B. 1991. V. 257. P. 119-125.

[30] Freund P. G. O., Zabrodin A. A hierarchic array of integrable models // J. Math. Phys. 1993. V. 34. № 12. P. 5832-5842.

[31] Сарнак П. Модулярные формы и их приложения. М.: Фазис, 1998.

[32] Pizer A. Ramanujan graphs and Hecke operators // Bull. Amer. Math. Soc. 1990. V. 23. № 1. P. 127-157.

[33] Erdös P. Graph theory and probability // Canad. J. Math. 1959. V. 11. P. 34-38.

[34] Romanov R. V., Rudin H. E. Scattering on the Bruhat-Tits tree. II. $p$-adic authomorphic functions // Препринт. С.-Петербург: СПбГУ, 1994.

[35] Harish-Chandra. Spherical functions on a semisimple Lie group. I, II // Amer. J. Math. 1958. V. 80. P. 241-310, 553-613.

[36] Гиндикин С. Г., Карпелевич Ф. И. Мера Планшереля римановых симметричных пространств неположительной кривизны // Докл. АН СССР. 1962. Т. 145. С. 252-255.

[37] Stark H. M., Terras A. A. Zeta functions of finite graphs and coverings // Adv. Math. 1996. V. 121. P. 124-165.

[38] Foata D., Zeilberger D. A combinatorial proof of Bass' evaluations of the Thara-Selberg zeta function for graphs // math.CO/9806037. (To appear in Trans. Amer. Math. Soc.)

[39] Romanov R. V., Rudin H. E. Scattering on p-adic graphs // Comput. Math. Appl. 1997. V. 34. № 5-6. P. 587-597.

[40] Chekhov L. L-functions in scattering on generalized Cayley trees // J. Tech. Phys. 1996. V. 37. P. 301-305. 
[41] Kashaev R. M. Quantization of Teichmüller spaces and the quantum dilogarithm // Preprint q-alg/9705021.

[42] Strebel K. Quadratic Differentials. Berlin: Springer-Verlag, 1984.

[43] Parry W., Pollicott M. An analogue of the prime number theorem for closed orbits of Axiom A flows // Ann. Math. 1983. V. 118. P. 573-591.

[44] Bedford T., Keane M., Series C. (Eds). Ergodic Theory, Symbolic Dynamics and Hyperbolic Spaces. Oxford: Oxford Univ. Press, 1991.

[45] Epstein C. L. Some exact trace formulas // Adv. Stud. Pure Math. 1992. V. 21. P. 127-140.

[46] McShane G. Simple geodesics and a Series constant over Teichmüller space // Invent. Math. 1998. V. 132. P. 607-632.

[47] Новиков С. П., Шварц А. С.Дискретные лагранжевы системы на графах. Симплекто-топологические свойства // УМН. 1999. Т. 54. № 1. С. 257-258.

[48] Akkermans E., Comtet A., Desbois J., Montambaux G., Texier C. Spectral determinants on quantum graphs // Preprint cond-mat/9911183.

Математический институт

Поступила в редакцию

им. В. А. Стеклова РАН

09.11.1999

E-mail: chekhov@mi.ras.ru 